\title{
Corrosion Rate Measurements Probabilistic Study of Chloride- Induced Acceleration Steel in Concrete Structures using Electrochemical Method
}

\author{
Charles Kennedy ${ }^{1}$, Philip Kpae. F. O ${ }^{2}$, Macmammah Michael ${ }^{3}$ \\ ${ }^{1}$ School of Engineering, Department of Civil Engineering, Kenule Beeson Saro-Wiwa \\ Polytechnic, Bori, Rivers State, Nigeria \\ ${ }^{2,3}$ School of Engineering, Department of Electrical /Electronics Engineering, Kenule Beeson \\ Saro-Wiwa Polytechnic, Bori, Rivers State, Nigeria
}

Authors E-mail: ${ }^{3}$ ken_charl@yahoo.co.uk, ${ }^{2}$ philipkpae1@gmail.com,

${ }^{3}$ Macmammahm@yahoo.com,

ABSTRACT
The research investigated corrosion potential probability assessment through half cell potential corrosion measurement, concrete resistivity and mechanical properties of reinforcing steel tests of non-corroded, corroded and inhibited reinforcement for and induced corrosion initiation process of an exposed concrete slab for 360 days. The results of controlled and coated samples with concrete resistivity obtained the maximum average value of $12.53 \mathrm{k} \Omega \mathrm{cm}$ and $15.51 \mathrm{k} \Omega \mathrm{cm}$ with a description of the value $10<\rho<20$ (low) compared to the corrosion value of $8.59 \mathrm{k} \Omega \mathrm{cm}$ with a description of $5<\rho<10$ ( high). The maximum yields obtained from the controlled and coated samples are $-110.11 \mathrm{mV}$ and $-123.04 \mathrm{mV}$, which showed the relationship between corrosion potential and probability asEcorr > $-200 \mathrm{mV}$ as the reference range. The results of this potential $E_{\text {corr }}$ result show that the controlled sample values and exudates/resin coated are low with a $90 \%$ probability that no corrosion of the reinforcement is observed at the time of measurement (10\% corrosion risk, $10 \%$ or shows an uncertain corrosion probability and for the samples of uncoated, the maximum computed value is $-331.55 \mathrm{mV}$, the result is within the reference value of the dependence between corrosion potential and probability of the value $-350 \mathrm{mV} \leq E$ corr $\leq$ $-200 \mathrm{mV}$ indicates a high-value range. From the results obtained it can be compared that the effect of corrosion attack was observed in the uncoated samples, while the samples with exudate/resin coating had corrosion protection properties with a highly resistant and waterresistant membrane that prevented corrosion of the reinforcing steel constructed into the concrete preventing slabs and induced accelerations from being exposed to corrosive media. The computed maximum percentage value of the controlled yield strength was $8.53 \%$ compared to the corroded and coated values of $-7.64 \%$ and $8.32 \%$ and the possible difference values of $0.05 \%$ controlled, $0.04 \%$ corroded and $0.05 \%$ coated. The maximum computed ultimate tensile strength percentage for comparison controlled $-6.61 \%$ versus corroded $6.68 \%$ and closed $-6.23 \%$, and different peaks controlled $0.06 \%$, corroded $0.07 \%$, and coated $0.06 \%$ and attacks on the uncoated (corroded) elements, which damage the reinforcing steel fibers, ribs, and passive formation and surface modification. The observed averagevalues for the coated samples were associated with the corrosion resistivity potential to penetrate the reinforcing steel with the formation of a protective membrane. The decrease in average and percentilesvalues indicated that the corrosion effect led to a reduction in diameter and crosssectional area, fiber degradation, rib reduction, and surface modification, whereas elements coated with exudate/resin confirmed an increase in volume as the layer thickness difference. 
For comparison, the results obtained show a reduction/reduction and reduction of average and percentile values for coatings with $0.955 \mathrm{~kg}$ to $0.835 \mathrm{~kg}$ and $14.44 \%$ to $-12.6 \%$ corroded. The combined results show that the corrosion effect on the corroded samples causes a decrease/decrease in weight compared to coatings with percentile loading and an increase in averagevalues, which causes a slight increase in volume with coating thickness. This study shows the efficacy and effectiveness of exudate/resin as an inhibitor against the effects of corrosion on reinforcement embedded in samples of concrete slabs exposed to induced corrosion.

Key Words: Corrosion, Corrosion inhibitors, corrosion potential, concrete resistivity and Steel Reinforcement

\section{INTRODUCTION}

Corrosion rates provide information on local corrosive conditions and the most effective corrosion prevention measures. Corrosion measurements provide early warning of damage in the process leading to corrosion induced failure. The high electrical resistivity of concrete reduces corrosion current and reduces corrosion rate. Surface resistivity of concrete is used to assess the corrosion rate of concrete and the ability of concrete to prevent corrosion. The cement matrix microstructure, pore structure, porosity and pore size distribution correlate with electrical resistivity. The electrical resistivity of concrete increases over time due to an increase in the degree of hydration of the cement-concrete paste. Usually, the electrical resistivity of concrete varies between 1 and $10,000 \mathrm{k} \Omega \mathrm{cm}$, depending on the moisture content of the concrete, the temperature and the quality of the concrete (composition, type of cement, etc.) [1]. In concrete, composite materials that form the microstructure and connect the pores to each other have a strong influence on durability. The higher the resistivity, the lower the corrosion current between the anode and cathode regions in reinforced concrete structures.

Measurement of half-cell potential is an indirect method for estimating the corrosion potential of corrosion, but recently much interest has been in developing tools to perform electrochemical measurements of disturbances in the steel itself to obtain direct estimates of corrosion rates [2]. Corrosion rate refers to electrochemical measurements based on data first reported by [3]. If potential measurements indicate a high probability of active corrosion, concrete resistivity measurements can be used to assess the degree of corrosion. This is also demonstrated in practice ([4] and [5]). Corrosion-related effects and destruction of reinforcing steel are limited by the development of corrosion inhibitors based on organic compounds with nitrogen, oxygen, sulfur atoms and double bonds in the molecule, which facilitate adsorption on metal surfaces [6].

Determination of corrosion rate by measuring weight loss in samples is still in use because it is simple and effective in some cases. However, the weight loss only gives the average corrosion of the entire metal sample over the entire test period. The corrosion effect of reinforcing embedded steel in the harsh and salty water environment is protected by the passive layer. As a result of corrosion, the surface is relatively uniform, but the specific properties of the metal surface are attacked. The environment is very susceptible to chlorideinduced reinforcement corrosion due to its high presence by loss of alkalinity due to chloride attack or concrete carbonation [7].This phenomenon leads to an increase in the mechanisms controlling these factors used inhibitors,

Corrosion of reinforcing steel embedded in reinforced concrete structures causes a reduction in the diameter of steel reinforcement, as a result of which mechanical properties such as 
tensile strength, tensile strength and ductility are reduced. Thus, the corrosion products take up a much larger volume than the original steel and end up exerting a large tensile force on the surrounding concrete, leading to cracking and falling of the concrete roof and loss of bonding strength between the steel and concrete. Measurement of half-cell potential is an indirect method for estimating the corrosion potential of corrosion, but recently much interest has been in developing tools to perform electrochemical measurements of disturbances in the steel itself to obtain direct estimates of corrosion rates [8]

[9] Stated that the passive potential range is very wide for steel and is usually from +200 $700 \mathrm{mV}$ saturated calomel electrode $(\mathrm{SCE})$ at $\mathrm{pH}=13$. Positives greater than $+200 \mathrm{mV}$ SCE cause oxygen evolution on passive steel. The evolution of oxygen causes a decrease in $\mathrm{OH}$-concentration at the steel / concrete interface. The evolution of oxygen causes a pool of water at the steel-concrete interface and therefore decreases the local resistivity. Under these conditions the corrosion rate is $0.04 \mathrm{mpy}$ at the steel surface, which is high for the passive state with oxygen reduction.

[10] Studied and examined the corrosion rate of embedded reinforcement in concrete ceiling structures that have been immersed in an uncomfortable environment and accelerated by Wenner's four drilling methods. The results showed a high final yield of corroded specimens for control samples and coated specimens due to the effect of corrosion on the mechanical properties of reinforcing steel. The results of the weight loss of steel, corroded sample showed a higher percentage compared to the control sample and the coated sample due to the corrosive effect on the mechanical properties of the steel. The results of the reduction of the cross-sectional area, corroded showed a higher percentile reduction value due to the effect of corrosion on the mechanical properties of the steel.

[11] Examined the passive loss of steel reinforcement with natural mineral exudates/resin from Milicia Excelsa with layer thicknesses of $150 \mu \mathrm{m}, 300 \mu \mathrm{m}$ and $450 \mu \mathrm{m}$. Coated and uncoated elements are inserted into concrete slabs and immersed in a fast flowing medium with fast application currents from $1200 \mathrm{mV}$ to $-200 \mathrm{mV}$, coated with a scan speed of $1 \mathrm{MV}$ for uncoated and half the scanning speed. Half cell corrosion potential, concrete resistivity and tensile strength. The result of the reduction in the cross section of the uncoated sample causes a higher corrosion value because of the effect of corrosion on the mechanical properties of reinforcing steel. Due to fiber / ribbed removal from the surface attack and the effect of corrosion on the mechanical properties of steel, the non-coated members showed higher percentage values against the control and coating samples. High-end yield and coating patterns of non-coated specimens with low load application to control lead to corrosion on the mechanical properties of steel reinforcement.

[12] Investigated the environmentally friendly inorganic content of Olibanum exudates/resins, coated with steel and that of non-coated members, embedded in a concrete slab and incubated in a corrosive environment for 150 days, with a test flow rate of $1200-200$ $\mathrm{mV}$ compared to control samples. Due to the attack on the mechanical properties of steel reinforcement, high yield results have been recorded for coated (corrugated) samples as opposed to coated specimens. The results of the weight loss of steel represent a higher percentage of values against the control and coating model, which results in a reduction in the fiber / ribbed properties of the steel and thus the surface strength. The cross-sectional expansion of the corrugated specimen results showed high percentage reduction values due to the effect of corrosion on the mechanical properties of the steel.

Novokshcheov [13] investigated and demonstrated that calcium nitrite does not affect the properties of concrete in any way, as shown by the problem of sodium or potassium-based inhibitors. A recent study by Skotinck [14] and Slater [15] showed that calcium nitrite showed better strength in terms of strength in accelerated long-term tests. 
[16] Investigated corrosion level probability assessment potential through half cell potential corrosion measurement, concrete resistivity test and tensile strength test mechanical properties of non-corroded, corroded and inhibited reinforcement with Moringa Oleifera lam resin paste of trees extract. When compared to corroded samples, corroded have showed increased values of potential Ecorr, $\mathrm{mV}$ and decreased values of concrete resistivity. Results of computed percentile average values of yield stress against ultimate strength, when compared to corrode as $100 \%$ nominal yield stress decreased from $105.75 \%$ to $96.12 \%$ and weight loss at $67.5 \%$ against $48.5 \%$ and $48.34 \%$ to $94.82 \%$, cross-sectional diameter reduction, both showed decreased values of corroded compared to coated specimens.

\section{MATERIALS AND METHODS FOR EXPERINMENT \\ Experimental Program \\ Aggregates}

Fine and coarse aggregates are purchased at the sand dumpsite. Both meet the requirements of [17]

\section{Cement}

Cement (lime) 42.5 was used for all concrete mixes. Cement meets the requirements of [18]

\section{Water}

Water samples were taken from the Civil Engineering Laboratory at Bori, Kenule Beeson Polytechnic in River State. Water meets [19] requirements

\section{Structural Steel Reinforcement}

Reinforcement directly purchased from the market in Port Harcourt. It conformed to [20] requirements

\section{Corrosion Inhibitors (Resins / Exudates) Gongronema latifolium}

The exudates were obtained from the stem and yield milky gummy exudates. They are abundantly seen in the Southern part of Nigeria. They are obtained from Chokocho Town in Etche Local Government of Rivers State

\section{Prepare samples for reinforcement with coated}

Obtained exudate/resin was directly coated to reinforcing steel of varying coating thicknesses embedded in the concrete slabs and exposed to coastal marine areas with high salinity concentration. Naturally, corrosion manifestation in reinforcement, metals and related materials is a long-term process that takes years. However, the artificial introduction of sodium chloride $(\mathrm{NaCl})$ speeds up the corrosion rate and manifestation occur in a short time. The rate of corrosion values is computed by estimating the current density that is gotten or obtained from the polarization curve and the quantification rate of corrosion level. Concrete mixes were batched by material weight with the manual mixing method using a standard concrete ratio of 1.2 .4 , and a water-cement ratio of 0.65 . The concrete slab of $100 \mathrm{~mm} \times 500 \mathrm{x}$ $500 \mathrm{~mm}$ (thickness, width, and length) with a concrete cover of $10 \mathrm{~mm}$ were cast into a metal mold, compacted to removed air, and reinforced with 10 numbers of $12 \mathrm{~mm}$ diameter reinforcing steel spaced at $100 \mathrm{~mm} \mathrm{c} / \mathrm{c}$ (top and bottom) and de-molded after 72 hours, cured for 28 days at standard room temperature to a hardened state. The hardened concrete slab was wholly immersed and a solution of $5 \%$ sodium chloride $(\mathrm{NaCl})$ to water and accelerated for rapid corrosion process for 360 days on interval checks and routine testing at 90 days, 180 days, 270 day, and 360 days with reading and records documentation for comparisons.

\section{Accelerated Corrosion Test}

The process of corrosion is a natural phenomenon that takes decades to manifest, that's a long-term process, but the rapid and accelerated corrosion process of using artificial 
substances of sodium chloride $(\mathrm{NaCl})$ allows the corrosion of reinforcing bars embedded in concrete, and can simulate the increase in corrosion that will occur over decades with a short period. To test the corrosion resistivity of concrete, an experimental process must be designed that will speed up the corrosion process and maximize the corrosion resistivity of the concrete. The accelerated corrosion test is an embossed current technique, an effective technique for examining the corrosion processes of steel in concrete and for assessing damage to the concrete cover. The laboratory acceleration process helps distinguish the role of individual factors that can influence chloride-induced corrosion. Therefore, for the design of structural elements and corrosion resistivity, as well as for the selection of suitable materials and a suitable protective system, it is necessary to carry out accelerated corrosion tests to obtain both quantitative and qualitative information about corrosion.

\section{Corrosion current measurement (Half - Cell Potential Measurement)}

Classification of the severity of corrosion of reinforcing steel is shown in Table 2.1. If the potential measurement results indicate a high probability of active corrosion, the degree of corrosion can be assessed by measuring the resistivity of the concrete. However, caution should be exercised when using these data, as the corrosion rate is assumed to be constant over time. This has also been proven by practical experience [Figg and Marsden [21], Gower and Millard [22]. Measuring half potential is an indirect method of estimating the probability of corrosion. Recently there has been great interest in the development of instruments for the electrochemical measurement of disturbances in steel itself to obtain direct estimates of corrosion rates (Stem and Geary [23]). Corrosion rates are related to electrochemical measurements, the first to be based on data.

Table 2.1: Dependence between potential and corrosion probability [24]

\begin{tabular}{|c|c|}
\hline Potential $E_{\text {corr }}$ & Probability of Corrosion \\
\hline $\begin{array}{l}\text { Ecorr }< \\
-350 \mathrm{mV}\end{array}$ & $\begin{array}{l}\text { Greater than } 90 \% \text { probability that reinforcing steel corrosion is occurring in } \\
\text { that area at the time of measurement }\end{array}$ \\
\hline $\begin{array}{ll}-350 \mathrm{mV} & \leq \\
E \mathrm{c}_{\text {orr }} & \leq \\
-200 \mathrm{mV} & \end{array}$ & Corrosion activity of the reinforcing steel in that area is uncertain \\
\hline $\begin{array}{l}E_{\text {corr }}> \\
-200 \mathrm{mV}\end{array}$ & $\begin{array}{l}90 \% \text { probability that no reinforcing steel corrosion is occurring in that area at } \\
\text { the time of measurement }(10 \% \text { risk of corrosion }\end{array}$ \\
\hline
\end{tabular}

\section{Test for measuring the resistivity of concrete}

Different readings are made at different points on the concrete surface. After the water has been applied to the slab surface, the resistivity of the concrete is measured daily at the reference point to determine its saturation state. The level of slab saturation is monitored by measuring the electrical resistivity of the concrete, which is directly related to the moisture content of the concrete. The time limit is a major challenge for all experimental measurements as the saturation state of the concrete changes over time. For this purpose, the four probes are touched directly to the concrete on the reinforcing steel rail. Before water is applied to the slab, the electrical resistivity of the concrete is measured at certain points in the dry state. The electrical resistivity becomes constant as soon as the concrete reaches saturation. 


\begin{tabular}{|l|l|}
\hline \hline \multicolumn{2}{|c|}{ Table 2.2: Dependence between concrete resistivity and corrosion probability [25] } \\
\hline Concrete resistivity $\boldsymbol{\rho}, \mathbf{k} \boldsymbol{\Omega} \mathbf{c m}$ & Probability of corrosion \\
\hline$\rho<5$ & Very high \\
\hline $5<\rho<10$ & High \\
\hline $10<\rho<20$ & Low to moderate \\
\hline$\rho>20$ & Low \\
\hline
\end{tabular}

\section{Tensile Strength of Reinforcement Bars}

To determine the yield and tensile strength of the bar, the reinforced, non-coated, and reinforced steel strip with a diameter of $12 \mathrm{~mm}$ was tested under stress in the Universal Test Machine (UTM) and subjected to direct stress until failure load is recorded. To ensure stability, the remaining cut pieces were used in subsequent bonding testing.

\section{EXPERIMENTAL RESULTS AND DISCUSSION}

The results of the half-cell potential measurements in Table 1 are plotted against the resistivity in Table 3 for easier interpretation. It is used as an indication of the probability of significant corrosion $(\rho<5,5<\rho<10,10<\rho<20, \rho>20)$ for very high, high, low to a moderate and low probability of corrosion. At another measurement point, the potential for $E_{\text {corr }}$ is high $\left(-350 \mathrm{mV} \leq E_{\text {corr }} \leq-200 \mathrm{mV}\right)$, which indicates a corrosion probability of $10 \%$ or uncertain. The results of concrete resistivity measurements are shown in Table 2. Concrete resistivity is usually measured using the four-electrode method.

Table 3.1: Potential Ecorr, after 28 days curing and 360days Accelerated Periods of Control Concrete slab Specimens

\begin{tabular}{|c|c|c|c|c|c|c|c|c|c|c|c|c|}
\hline \multirow[b]{2}{*}{ Sumple Numbers } & \multicolumn{12}{|c|}{ Control Concrete slab Specimens } \\
\hline & GLS & GLSI & GLS2 & GLS3 & GLS4 & GLS5 & GLS6 & GLS7 & GLS8 & GLS9 & GLSI0 & GLSII \\
\hline & \multicolumn{12}{|c|}{ Time Interval after 28 days curing } \\
\hline $\begin{array}{l}\text { Sampling and } \\
\text { Durations }\end{array}$ & \multicolumn{3}{|c|}{ Samples 1 (28 days) } & \multicolumn{3}{|c|}{ Samples 2 (28 Days) } & \multicolumn{3}{|c|}{ Samples 3 (28 Days) } & \multicolumn{3}{|c|}{ Samples 4 (28 Days) } \\
\hline Potential Ecorr,mV & 114.84 & 113.52 & 109.25 & 11285 & 110.26 & 107.23 & $\overline{115.68}$ & 111.36 & $\overline{106.91}$ & 114.22 & 113.21 & 112.37 \\
\hline $\begin{array}{c}\text { Concrete Resistivity } \rho \text {, } \\
\text { kSacm }\end{array}$ & 12.40 & 12.39 & 12.39 & 12.38 & 12,37 & 12.54 & 12.53 & 12,53 & 12.52 & 12.51 & 12.46 & 12,37 \\
\hline $\begin{array}{l}\text { Yield Strength, fy } \\
(\mathrm{MPa})\end{array}$ & 455,53 & 458,53 & 454.53 & 454.83 & 455.53 & 454.76 & 457.76 & 458.06 & 456.76 & 458.15 & 454.66 & 458.49 \\
\hline $\begin{array}{l}\text { Ultimate Tensile } \\
\text { Strength, fu (MPa) }\end{array}$ & 026.78 & 624,73 & 626.41 & 622.19 & 625.72 & 626.14 & 625.94 & 626.74 & 625.34 & 626.89 & 626.39 & 626.25 \\
\hline Strain Ratio & 1.38 & 1.36 & 1.38 & 1.37 & 1.37 & 1.38 & 1.37 & 1.37 & 1.37 & 1.37 & 1.38 & 1.37 \\
\hline $\begin{array}{l}\text { Rebur Diameter } \\
\text { Before Test (mm) }\end{array}$ & 11.96 & 11.96 & 11.97 & 11.96 & 11.96 & 11.97 & 11.97 & 11.95 & 11.96 & 11.96 & 11.96 & 11.97 \\
\hline $\begin{array}{c}\text { Rebar Diamete at } 28 \\
\text { days }(\mathrm{mm})\end{array}$ & 11.96 & 11.96 & 11.97 & 11.96 & 11.96 & 11.97 & 11.97 & 11.95 & 11.96 & 11.96 & 11.96 & 11.97 \\
\hline $\begin{array}{l}\text { Cross- sectional Area } \\
\text { Reduction/Increase ( } \\
\text { Diameter, mm) }\end{array}$ & 0.00 & 0.00 & 0.00 & 0.00 & 0.00 & 0.00 & 0.00 & 0.00 & 0.00 & 0.00 & 0.00 & 0.00 \\
\hline $\begin{array}{l}\text { Rebar Weights- } \\
\text { Bcfore Test }\end{array}$ & 0.88 & 0.88 & 0.88 & 0.88 & 0.88 & 0.88 & 0.88 & 0.88 & 0.88 & 0.88 & 0.89 & 0.88 \\
\hline $\begin{array}{l}\text { Rebar Weights-After } \\
\text { at } 28 \text { days }(\mathrm{Kg})\end{array}$ & 0.88 & 0.88 & 0.88 & 0.88 & 0.88 & 0.88 & 0.88 & 0.88 & 0.88 & 0.88 & 0.89 & 0.88 \\
\hline $\begin{array}{l}\text { Weight Loss / Gain of } \\
\text { Steel (Kg) at } 28 \text { days }\end{array}$ & 0.00 & 0.00 & 0.00 & 0.00 & 0.00 & 0.00 & 0.00 & 0.00 & 0.00 & 0.00 & 0.00 & 0.00 \\
\hline
\end{tabular}


DOI : https://dx.doi.org/10.26808/rs.st.i11v4.04

International Journal of Advanced Scientific and Technical Research

ISSN 2249-9954

Available online on http://www.rspublication.com/ijst/index.htm

Issue 11 volume 4 July- August 2021

Table 3.2: Potential Ecorr, after 28 days curing and 360days Accelerated Periods of Corroded Concrete slab Specimens

\begin{tabular}{|c|c|c|c|c|c|c|c|c|c|c|c|c|}
\hline Sampling and & \multicolumn{3}{|c|}{ Samples 1 ( 90 days) } & \multicolumn{3}{|c|}{ Samples 2 (180 Days) } & \multicolumn{3}{|c|}{ Samples 3 (270 Days) } & \multicolumn{3}{|c|}{ Samples 4 (360 Days) } \\
\hline Poteatial & - & - & - & 2 & - & - & - & - & - & - & - & - \\
\hline Ecorr, mV & 316,50 & 340.63 & 337.53 & 329.92 & 339.72 & 340.72 & 380,62 & 387.82 & 391.92 & 395.04 & 399.24 & 397.47 \\
\hline $\begin{array}{c}\text { Concrete } \\
\text { Resistivity } \rho \text {, } \\
\mathrm{k \Omega} \mathrm{cm}\end{array}$ & 8.20 & 8.38 & 8.21 & 8.22 & 9.00 & 8.55 & 8.17 & 8.73 & 8.76 & 8.36 & 8.54 & 8.55 \\
\hline $\begin{array}{l}\text { Yield Strength, fy } \\
\text { (MPa) }\end{array}$ & 419.77 & 422.77 & 418.77 & 419.07 & 419.77 & 419.00 & 422.00 & 422.30 & 421.00 & 422.39 & 418.90 & 422.73 \\
\hline $\begin{array}{l}\text { Ultimate Tensile } \\
\text { Strength, fu (MPa) }\end{array}$ & 618.72 & 616.67 & 618.35 & 614.13 & 617.66 & 618.08 & 617.88 & 618.68 & 617.28 & 618.83 & 61833 & 618.19 \\
\hline Strain Ratio & 1.47 & 1.46 & 1.48 & 1.47 & 1.47 & 1.48 & 1.46 & 1.47 & 1.47 & 1.47 & 1.48 & 1.46 \\
\hline $\begin{array}{l}\text { Rebar Diameter } \\
\text { Before Test ( } \mathrm{mm} \text { ) }\end{array}$ & 11.96 & 11.95 & 11.96 & 11.96 & 11.95 & 11.97 & 11.96 & 11.95 & 11.96 & 11.96 & 11.95 & 11.96 \\
\hline $\begin{array}{c}\text { Rebar Diameter- } \\
\text { After } \\
\text { Corrosion(mm) }\end{array}$ & 11.92 & 11.91 & 11.92 & 11.92 & $11: 91$ & 11.93 & 11.92 & 11.91 & 11.92 & 11.91 & 11.91 & 11.92 \\
\hline $\begin{array}{l}\text { Cross- sectional } \\
\text { Area } \\
\text { Reduction/Increase } \\
\text { ( Diameter, mm) }\end{array}$ & 0.05 & 0.05 & 0.05 & 0.05 & 0.05 & 0.05 & 0.05 & 0.05 & 0.05 & 0.05 & 0.05 & 0.05 \\
\hline $\begin{array}{l}\text { Rebar Weights- } \\
\text { Before Test }(\mathrm{Ky})\end{array}$ & 0.88 & 0.88 & 0.88 & 0.88 & 0.88 & 0.88 & 0.88 & 0.88 & 0.88 & 0.88 & 0.89 & 0.88 \\
\hline $\begin{array}{c}\text { Rebar Weights- } \\
\text { After } \\
\text { Corrosion(Kg) }\end{array}$ & 0.83 & 0.83 & 0.83 & 0.83 & 0.83 & 0.83 & 0.83 & 0.83 & 0.83 & 0.83 & 0.84 & 0.83 \\
\hline $\begin{array}{l}\text { Weight Loss /Gain } \\
\text { of Steel (Kg) }\end{array}$ & 0.05 & 0.05 & 0.05 & 0.05 & 0.05 & 0.05 & 0.05 & 0.05 & 0.05 & 0,05 & 0.05 & 0.05 \\
\hline
\end{tabular}

Table 3.3: Potential Ecorr, after 28 days curing and 360days Accelerated Periods of

Gongronema latifolium Exudate / Resin Coated Specimens

\begin{tabular}{|c|c|c|c|c|c|c|c|c|c|c|c|c|}
\hline Sampling and & \multicolumn{3}{|c|}{ Samples 1 (90 days) } & \multicolumn{3}{|c|}{ Samples 2 (180 Days) } & \multicolumn{3}{|c|}{ Samples 3 (270 Days) } & \multicolumn{3}{|c|}{ Samples 4 (360 Days) } \\
\hline & $150 \mu$ & $\begin{array}{l}\text { (Exudat } \\
\text { couted }\end{array}$ & Resin) & $300 \mu \mathrm{r}$ & $\begin{array}{l}\text { (Exudat } \\
\text { coated }\end{array}$ & Resin) & $450 \mu$ & $\begin{array}{l}\text { (Exudat } \\
\text { coated }\end{array}$ & Resin) & $600 \mu \mathrm{mi}$ & xudate'R & in) coated \\
\hline $\begin{array}{l}\text { Potential } \\
\text { Ecorr,mV }\end{array}$ & -124.43 & -128.11 & -12.3 .84 & -122.44 & 124.85 & -121.82 & -130.27 & 125.95 & -121.50 & $-123,81$ & -127.80 & -119.08 \\
\hline $\begin{array}{c}\text { Concrete } \\
\text { Resistivity p, } \\
\text { k } \Omega \mathrm{cm}\end{array}$ & 14.91 & 15.06 & 15.34 & 15.47 & 15.16 & 15.45 & 15.40 & 15.55 & 15.58 & 15.05 & 14.94 & 14.79 \\
\hline $\begin{array}{c}\text { Yield Strength, fy } \\
\text { (MPa) }\end{array}$ & 454.67 & 457.67 & 453.67 & 453.97 & 454.67 & 453.90 & 456.90 & 457.20 & 455,90 & 457.29 & 453.80 & 457.63 \\
\hline $\begin{array}{l}\text { Ultimate Tensile } \\
\text { Strength, fu (MPa) }\end{array}$ & 628.44 & 626.39 & 628,07 & 623.85 & 627.38 & 627.80 & 627.60 & 628.40 & 627.00 & 628.55 & 628.05 & 627.91 \\
\hline Strain Ratio & 1.38 & 1,37 & 1.38 & 1.37 & 1.38 & 1.38 & 1.37 & 1.37 & 1.38 & 1.37 & 1.38 & 1.37 \\
\hline $\begin{array}{l}\text { Rebar Diameter } \\
\text { Before Test (mm) }\end{array}$ & 11.96 & 11.95 & 11.96 & 11.96 & 11.95 & 11.97 & 11.96 & 11.95 & 11.96 & 11.96 & 11.95 & 11.96 \\
\hline $\begin{array}{l}\text { Rebar Diameter- } \\
\text { After } \\
\text { Corrosion(mm) }\end{array}$ & 12.02 & 12,01 & 12,02 & 12.02 & 12.01 & 12.03 & 12.02 & 12.01 & 12.02 & 12.02 & 12.01 & 12.02 \\
\hline $\begin{array}{c}\text { Cross- sectional } \\
\text { Area } \\
\text { Reduction/increase } \\
\text { (Diameter, mm) }\end{array}$ & 0.06 & 0.06 & 0.06 & 0.06 & 0,06 & 0.06 & 0.06 & 0.06 & 0.06 & 0,06 & 0.06 & 0.96 \\
\hline $\begin{array}{l}\text { Rebar Weights- } \\
\text { Before Test }(\mathrm{Kg})\end{array}$ & 0.88 & 0.88 & 0.88 & 0.88 & 0,88 & 0.88 & 0.88 & 0.88 & 0.88 & 0.88 & 0.89 & 0.88 \\
\hline $\begin{array}{c}\text { Rebar Weights- } \\
\text { After } \\
\text { Corrosion(Kg) }\end{array}$ & 0.95 & 0.95 & 0.95 & 0.95 & 0.95 & 0.95 & 0.95 & 0.95 & 0.95 & 0.95 & 0.96 & 0.95 \\
\hline $\begin{array}{c}\text { Weight Loss / Gain } \\
\text { of Steel (Kg) }\end{array}$ & 0.07 & 0.07 & 0.07 & 0.07 & 0.07 & 0.07 & 0.07 & 0.07 & 0.07 & 0.07 & 0.07 & 0.07 \\
\hline
\end{tabular}


Table 3.4: Average Potential Ecorr, after 28 days curing and 360days Accelerated Periods ( Control, Corroded and Exudate/Resin Coated (specimens)

\begin{tabular}{|c|c|c|c|c|c|c|c|c|c|c|c|c|}
\hline $\begin{array}{l}\text { Sampling and } \\
\text { Durations }\end{array}$ & \multicolumn{4}{|c|}{ Control Concrete slab Specimens } & \multicolumn{4}{|c|}{$\begin{array}{l}\text { Corroded Concrete slab } \\
\text { Specimens }\end{array}$} & \multicolumn{4}{|c|}{$\begin{array}{l}\text { Gongronema latifolium Exudate } \\
\text { /Resin Coated Specimens }\end{array}$} \\
\hline & \multicolumn{4}{|c|}{$\begin{array}{l}\text { Average Potential Ecorr, Values } \\
\text { of Control Concrete slab } \\
\text { Specimens }\end{array}$} & \multicolumn{4}{|c|}{$\begin{array}{l}\text { Average Potential Ecorr, Values } \\
\text { of Corroded Concrete slab } \\
\text { Specimens }\end{array}$} & \multicolumn{4}{|c|}{$\begin{array}{l}\text { Average Potential Ecorr. Values } \\
\text { of Gongronema latifolium } \\
\text { Exudate / Resin Coated } \\
\text { Specimens }\end{array}$} \\
\hline Potential Ecot & $\overline{12.53}$ & 110.11 & $11 \overline{1.31}$ & $11 \overline{3}, 26$ & $33 i .55$ & 338.78 & 386.78 & $39 \overline{7.25}$ & 125.46 & 123.04 & 125.91 & 123.57 \\
\hline $\begin{array}{r}\text { Concrete Re: } \\
p, \mathrm{k} \Omega \mathrm{c}\end{array}$ & 12.39 & 12.43 & 12.53 & 12.45 & 8.27 & 8.59 & 8.55 & 8.48 & 15.11 & 15.36 & 15.51 & 14.93 \\
\hline $\begin{array}{l}\text { Yield Strength, fy } \\
\text { (MPa) }\end{array}$ & 456.20 & 455.04 & 457.53 & 457.10 & 420.44 & 419.28 & 421.77 & 421.34 & 455.34 & 454.18 & 456.67 & 456.24 \\
\hline $\begin{array}{l}\text { Uhimate Tensile } \\
\text { Strength. fit (MPa) }\end{array}$ & 5.97 & 624.68 & 626.01 & 626.51 & 617.92 & 616.63 & 617.95 & 618.45 & 627.64 & 626.35 & 627.67 & 628.17 \\
\hline Strain Ratio & 37 & 1.37 & 1.37 & 1.37 & 1.47 & 1.47 & 1.47 & 1.47 & 1.38 & 1.38 & 1.37 & 1.38 \\
\hline $\begin{array}{l}\text { Rebar Di } \\
\text { Before Tes }\end{array}$ & 11.96 & 11.97 & 11.96 & 11.96 & 11.96 & 11,96 & 11.96 & 11.96 & 11.96 & 11.96 & 11.96 & 11.96 \\
\hline $\begin{array}{l}\text { Rebar Diameter- } \\
\text { After Corrosion(min) }\end{array}$ & 11.96 & 11.97 & 11.96 & 11.96 & 1.92 & 11.92 & 11.91 & 11.91 & 12.02 & 12.02 & 12.02 & 12.02 \\
\hline $\begin{array}{l}\text { Cross- sectional Area } \\
\text { Reduction/Increase ( } \\
\text { Diameter, mm) }\end{array}$ & 0.00 & 0.00 & 00 & 0.00 & 052 & 0.057 & 0.053 & 0.059 & 0.069 & 0.068 & 0.065 & 0.071 \\
\hline $\begin{array}{l}\text { Rebar Weights- } \\
\text { Bcfore Test }(\mathrm{Kg})\end{array}$ & 0.882 & 0.885 & 0.888 & 0.883 & 0.891 & 0.888 & 0.88 & 0.891 & 0.889 & 0.885 & 0.88 & 0.891 \\
\hline $\begin{array}{l}\text { Rebar Weights- After } \\
\text { Corrosion }(\mathrm{Kg})\end{array}$ & 0.88 & 0.88 & 0.88 & 0.88 & 0.83 & 0.835 & 0.832 & 0.831 & 0.945 & 0.952 & 0.955 & 0,945 \\
\hline $\begin{array}{l}\text { Weight Loss / Gain of } \\
\text { Steel (Kg) }\end{array}$ & 0.00 & 0.00 & 0 & 0 & 1 & 57 & 0.059 & 52 & 51 & 0.079 & 0.078 & 0.071 \\
\hline
\end{tabular}

Table 3.5: Average Percentile Potential Ecorr, after 28 days curing and 360days Accelerated Periods ( Control, Corroded and Exudate/Resin Coated (specimens)

\begin{tabular}{|c|c|c|c|c|c|c|c|c|c|c|c|c|}
\hline & \multicolumn{4}{|c|}{$\begin{array}{l}\text { Control Concrete slab } \\
\text { Specimens }\end{array}$} & \multicolumn{4}{|c|}{$\begin{array}{l}\text { Corroded Concrete slab } \\
\text { Specimens }\end{array}$} & \multicolumn{4}{|c|}{$\begin{array}{c}\text { Gongronema latifolium } \\
\text { Exudate / Resin Coated } \\
\text { Specimens. }\end{array}$} \\
\hline & $\begin{array}{l}\text { Percer } \\
\text { Econ } \\
\text { Con }\end{array}$ & $\begin{array}{l}\text { e Av } \\
\text { Value } \\
\text { te sla }\end{array}$ & $\begin{array}{l}\text { ige } P \\
\text { of } C \\
\text { Spoci }\end{array}$ & $\begin{array}{l}\text { atial } \\
\text { rol } \\
\text { ns }\end{array}$ & $\begin{array}{l}\text { Pere: } \\
\text { Eco } \\
\text { Col }\end{array}$ & $\begin{array}{l}\text { tile Av } \\
\text { Valuc } \\
\text { rete sl }\end{array}$ & of $\mathrm{Co}$ & $\begin{array}{l}\text { itial } \\
\text { led }\end{array}$ & $\begin{array}{l}\text { Percet } \\
\text { Got } \\
\text { Ext }\end{array}$ & $\begin{array}{l}\text { titile Av } \\
\text { Ecorr. } \\
\text { ugroner } \\
\text { date / } \\
\text { Sped }\end{array}$ & $\begin{array}{l}\text { crage P } \\
\text { alues } \\
\text { a Iatifo } \\
\text { esin Co } \\
\text { mens }\end{array}$ & $\begin{array}{l}\text { tential } \\
\text { ium } \\
\text { ated }\end{array}$ \\
\hline Potential Ecorr,mV & $-\overline{66.06}$ & 67.50 & 71.22 & 71.49 & 164.26 & 175.34 & 207.19 & 221.49 & 62.16 & 63.68 & 67.45 & 68.90 \\
\hline $\begin{array}{c}\text { Concrete Resistivity } \\
\text { p, knem }\end{array}$ & 49.95 & 44.73 & 46.46 & 46.79 & -45.29 & -44.09 & -44.87 & $-43,19$ & 82.77 & 78.85 & 81,38 & 76.03 \\
\hline $\begin{array}{c}\text { Yield Strength, fy } \\
(\mathrm{MPa})\end{array}$ & 8.51 & 8.53 & 8.48 & 8.40 & -7.66 & -7.68 & -7.64 & -7.65 & 8.30 & 8.32 & 8.27 & 8.28 \\
\hline $\begin{array}{l}\text { Ultimate strength } \\
\qquad(\mathrm{N} / \mathrm{mm} 2)\end{array}$ & 1.30 & 1.31 & 1.30 & 1.30 & -1.55 & -1.55 & -1.55 & -1.55 & 1.57 & 1.58 & 1.57 & 1.57 \\
\hline Strain Ratio & 67 & 66 & 2 & 1 & 68 & 67 & 67 & 6.61 & 6.26 & 6.25 & 6.21 & -6.20 \\
\hline $\begin{array}{l}\text { Rebar Diame } \\
\text { Before Test in }\end{array}$ & +16 & 8 & & & & 0 & & 7 & 18 & & 418 & 0.411 \\
\hline $\begin{array}{l}\text { Rebar Diameter- } \\
\text { After } \\
\text { Corrosion(mm) }\end{array}$ & 0.416 & 0.418 & 0.419 & 0.416 & -0.897 & -0.879 & $-0,985$ & -0.978 & 0.128 & 0.138 & 0.139 & 0,142 \\
\hline $\begin{array}{c}\text { Cross-sectional } \\
\text { Arca } \\
\text { Reduction/Increase ( } \\
\text { Diameter, mm) }\end{array}$ & 0.00 & 0.00 & 0.00 & 0.00 & -22.03 & $-22,03$ & -22.03 & -22.03 & 28,26 & 28.26 & 28.26 & 28.26 \\
\hline $\begin{array}{l}\text { Rebar Weights- } \\
\text { Before Test }(\mathrm{Kg})\end{array}$ & 9.78 & 9.99 & 9.99 & 9.87 & -17.92 & -15.6 & -15.6 & -15.6 & 17.13 & 13.93 & 13.93 & 13.93 \\
\hline $\begin{array}{l}\text { Rebar Weights- } \\
\text { After Corrosion( } \mathrm{Kg} \text { ) }\end{array}$ & 0.00 & 0.00 & 0.00 & 0.00 & -12.62 & -12.61 & -12.61 & $-12,59$ & 14.44 & 14.42 & 14.42 & 14.41 \\
\hline $\begin{array}{l}\text { Weight Loss / Gain } \\
\text { of Stecl (Kg) }\end{array}$ & 0.00 & 0.00 & 0.00 & 0.00 & -23.57 & -24.56 & -24.67 & -23.51 & 34,06 & 37.33 & 36.95 & 39,35 \\
\hline
\end{tabular}




\section{Results of Concrete Resistivity $\rho, \mathrm{k} \Omega \mathrm{cm}$ and Potential Ecorr, $\mathrm{mV}$, on Concrete Slab Members}

Half-cell potential measurement is an indirect method for estimating corrosion potential, but recently there has been much interest in the development of tools to perform perturbing electrochemical measurements on steels themselves to obtain direct estimates of corrosion rates [26]. Corrosion rates are related to electrochemical measurements based on data first reported by [27]. If potential measurements indicate a high probability of active corrosion, concrete resistivity measurements can be used to assess the degree of corrosion. The effect and damage of reinforcing steel by corrosion is limited by the development of corrosion inhibitors based on organic compounds with nitrogen, oxygen, sulfur atoms, and double bonds in the molecule, which facilitate adsorption on metal surfaces [28].

The results of Potential $\mathrm{E}_{\text {corr }}, \mathrm{mV}$ and Concrete Resistivity $\rho, \mathrm{k} \Omega \mathrm{cm}$ are obtained from Tables 3.1-3.3 and summarized into average and percentile values in Tables 3.4 and 3.5, plotted graphically in Figures 3.1-3.7b, are the results of controlled samples, non-coated (corroded) and coated for 36 concrete slabs, divided into 3 sets of 12 controlled samples, which is the determinant reference range, 12 non-coated (corroded) samples, and 12 exudate/resin coated samples.

The minimum and maximum values of concrete resistivity at average and percentile values with controlled sample potential difference were $12.39 \mathrm{k} \Omega \mathrm{cm}$ and $12.53 \mathrm{k} \Omega \mathrm{cm}(44.73 \%$ and $49.95 \%$ ) and the difference values were $0.14 \mathrm{k} \Omega \mathrm{cm}$ and $5.22 \%$, samples that corroded were $8.27 \mathrm{k} \Omega \mathrm{cm}$ and $8.59 \mathrm{k} \Omega \mathrm{cm}(-45.3 \%$ and $-43.2 \%)$, and the difference values were $0.32 \mathrm{k} \Omega \mathrm{cm}$ and $2.1 \%$., The coated samples value are $14.93 \mathrm{k} \Omega \mathrm{cm}$ and $15.51 \mathrm{k} \Omega \mathrm{cm}(76.03 \%$ and $82.77 \%)$ and the difference values were $0.58 \mathrm{k} \Omega \mathrm{cm}$ and $6.74 \%$, respectively.

The computed maximum percentile values for the concrete resistivity of the controlled sample resistivity were $49.95 \%$ versus the corroded and coated values $-43.2 \%$ and $82.77 \%$ and the maximum percentile difference values from the control were $5.22 \%$ versus the corroded and coated values were $2,1 \%$ and $6.74 \%$. The results of controlled and coated samples with concrete resistivity obtained the maximum average value of $12.53 \mathrm{k} \Omega \mathrm{cm}$ and $15.51 \mathrm{k} \Omega \mathrm{cm}$ with a description of the value $10<\rho<20$ (low) compared to the corrosion value of $8.59 \mathrm{k} \Omega \mathrm{cm}$ with a description of $5<\rho<10$ ( high) and with a reference range of dependence between concrete resistivity and corrosion probability at significant corrosion probability $(\rho<5,5<\rho<10,10<\rho<20, \rho>20)$ for very high, high, low to moderate, and low, for possible corrosion. From the comparison results of coated and corroded samples, the maximum values obtained for both samples clearly indicate the value of coated samples with a range of $10<\rho<20$, which classifies the range of values as low to moderate, with information as significant corrosion probability. The maximum value of the corroded sample was in the range of $5<\rho<10$, indicating high, signs indicating the presence of possible corrosion, as stated by ([10], [16], [12], [11]).

The average and percentile values of the minimum, maximum, and differential of the computed potential measurements from the half-cell potential of the controlled samples are $113.26 \mathrm{mV}$ and $-110.11 \mathrm{mV}(-71.5 \%$ and $-66.06 \%)$ with potential difference of $(3.15 \mathrm{mV}$ and $5.429 \%$ ), the corroded samples are $-397.25 \mathrm{mV}$ and $-331.55 \mathrm{mV}(164.2 \%$ and $221.49 \%)$ and differential values of $65.7 \mathrm{mV}$ and $57.23 \%$ and the coated samples are $-126.91 \mathrm{mV}$ and $123.04 \mathrm{mV}(-68.9 \%$ and $-62.2 \%)$ and potential difference are $2.87 \mathrm{mV}$ and $6.74 \%$ respectively. The maximum computed percentile control value is $-66.06 \%$ compared to the corroded and coated values of $221.49 \%$ and $-62.2 \%$ and the controlled potential difference value is $5.429 \%$, corroded $57.23 \%$, and coated $6.74 \%$. The maximum yields obtained from the controlled and coated samples are $-110.11 \mathrm{mV}$ and $-123.04 \mathrm{mV}$, which showed the relationship between corrosion potential and probability as $E$ corr $>-200 \mathrm{mV}$ as the reference range. The results of this potential $\mathrm{E}_{\text {corr }}$ result show that the controlled sample values and exudates/resin 
coated are low with a $90 \%$ probability that no corrosion of the reinforcement is observed at the time of measurement $(10 \%$ corrosion risk, $10 \%$ or shows an uncertain corrosion probability and for the samples of uncoated, the maximum computed value is $-331.55 \mathrm{mV}$, the result is within the reference value of the dependence between corrosion potential and probability of the value $-350 \mathrm{mV} \leq E$ corr $\leq-200 \mathrm{mV}$ indicates a high-value range, indicating a corrosion probability of $10 \%$ or uncertain from the reference range (controlled) shows that the corroded sample shows corrosion as a result of accelerated corrosion-induced as compared to the coated sample which does not show corrosion attack on reinforcing steel embedded in a concrete slab, exposed to the corrosive environment due to layer formation resistive.

From the results obtained it can be compared that the effect of corrosion attack was observed in the uncoated samples, while the samples with exudate/resin coating had corrosion protection properties with a highly resistant and water-resistant membrane that prevented corrosion of the reinforcing steel constructed into the concrete preventing slabs and induced accelerations from being exposed to corrosive media.

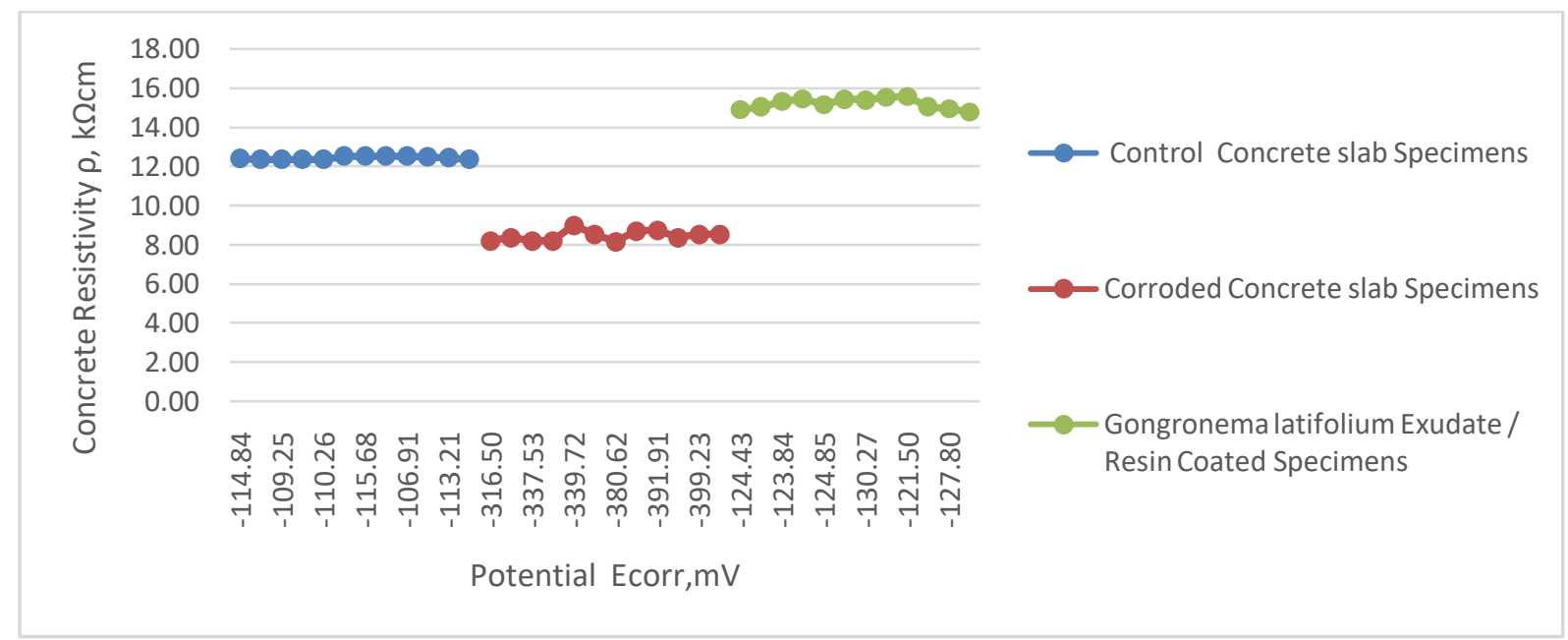

Figure 3.1 : Concrete Resistivity $\rho, \mathrm{k} \Omega \mathrm{cm}$ versus Potential Ecorr,mV Relationship

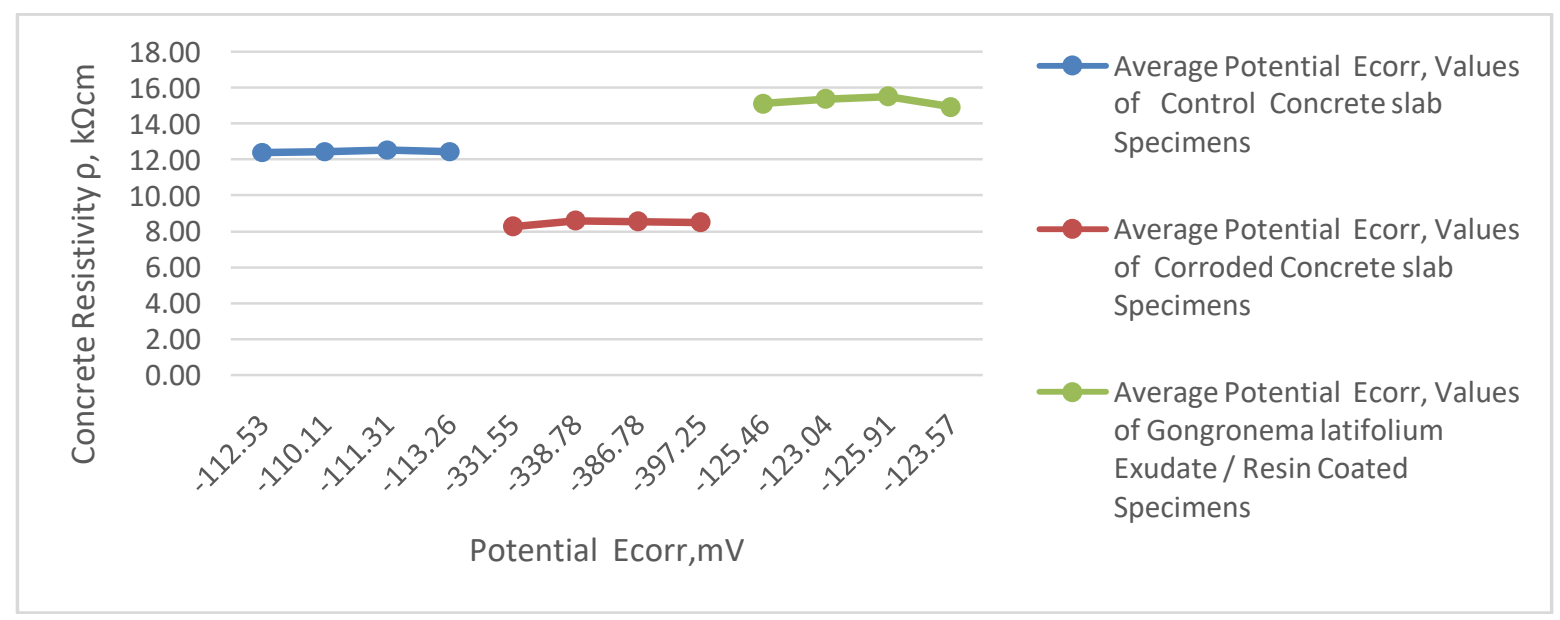

Figure 3.1A: Average Concrete Resistivity versus Potential Relationship 


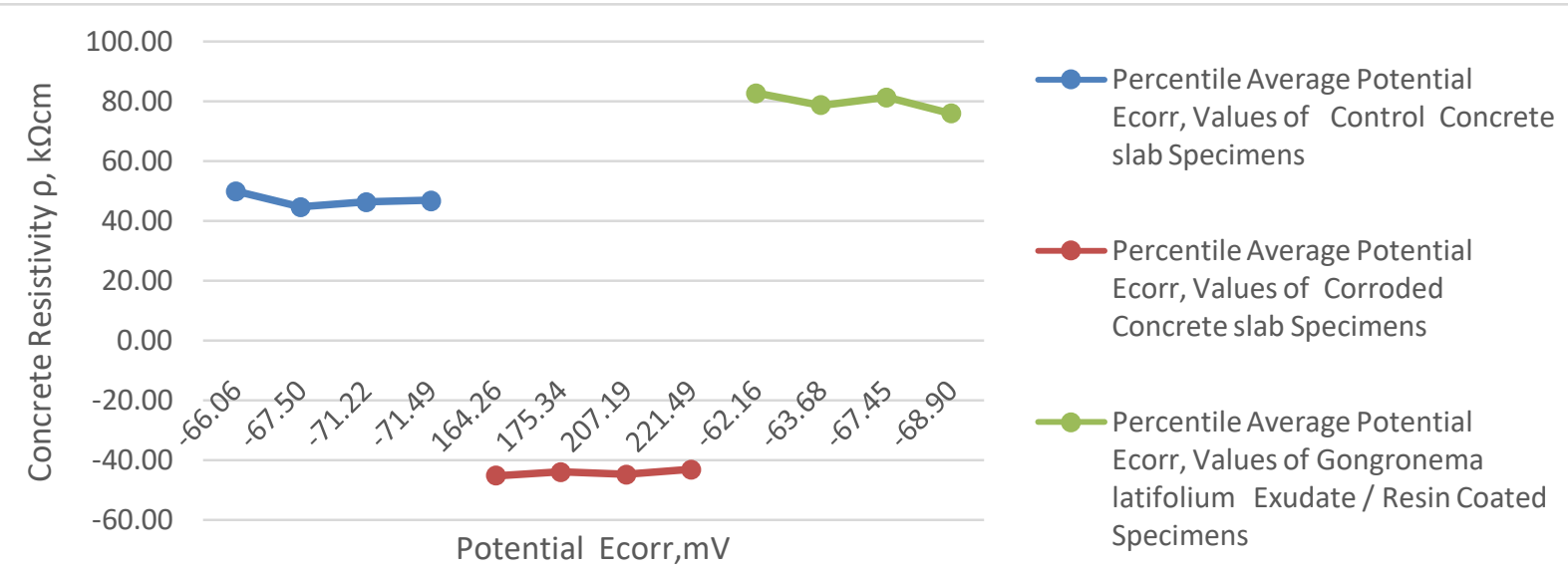

Figure 3.1B : Average Percentile Concrete Resistivity versus Potential Relationship

\section{Results of Mechanical Properties of Yield Strength, Ultimate Strength and Strain Ratio of Embedded Reinforcing Steel in Concrete Slab}

Corrosion of reinforcing steel embedded in reinforced concrete structures causes a reduction in the diameter of the steel reinforcement, which reduces mechanical properties such as tensile strength, tensile strength, and ductility. Thus, the corrosion products take up a much larger volume than the original steel and in the end exert a large tensile force on the surrounding concrete, which causes cracking and falling of the concrete roof and loss of the bond between steel and concrete. The results of the mean, percentile, and the difference between the minimum and maximum recovery limits, fy $(\mathrm{MPa})$ of the controlled sample were $455.04 \mathrm{MPa}$ and $457.53 \mathrm{MPa}(8.48 \%$ and $8.53 \%)$ and the difference values were $2.49 \mathrm{MPa}$ and $00.05 \%$, the corroded sample was $419.28 \mathrm{MPa}$ and $421.77 \mathrm{MPa}(-7.68 \%$ and $-7.64 \%)$ and the difference value was $2.49 \mathrm{MPa}$ and $0.04 \%$, the coated sample value was $454.18 \mathrm{MPa}$ and $456.67 \mathrm{MPa}(8.27 \%$ and $8.32 \%)$ and the difference value is $2.49 \mathrm{MPa}$ and $0.05 \%$. The computed maximum percentage value of the controlled yield strength was $8.53 \%$ compared to the corroded and coated values of $-7.64 \%$ and $8.32 \%$ and the possible difference values of $0.05 \%$ controlled, $0.04 \%$ corroded and $0.05 \%$ coated.

The mean, percentile, and the difference between the minimum and maximum ultimate tensile strength, fu (MPa) of the controlled sample were $624.68 \mathrm{MPa}$ and $626.51 \mathrm{MPa}(1.3 \%$ and $1.31 \%$ ) and the difference values were $1.83 \mathrm{MPa}$ and $0.01 \%$, corroded $616.6 \mathrm{MPa}$ and 618.5 $\mathrm{MPa}(-1.55 \mathrm{MPa}$ and $-1.55 \%)$ and the difference was $1.82 \mathrm{MPa}$ and $0.00 \%$, the coating was $626.35 \mathrm{MPa}$ and $628,17 \mathrm{MPa}(1.57 \%$ and $1.158 \%$ and the difference value is $1.82 \mathrm{MPa}$ and $0.01 \%$ ) The percentage value of the maximum tensile strength computed from the controlled is $1.31 \%$ relative to the corrosion and coating values $-1.55 \%$ and $1,57 \%$ and $0.01 \%$ possible difference value checked, $0.00 \% \%$ corroded and $0.01 \%$ coated.

The minimum and maximum averagevalues of the deformation ratio, percentile, and difference values of the controlled samples were 1.37 and $1.37(-6.67 \%$ and $-6.61 \%)$ with a difference of 0.00 and $0.06 \%$, respectively. the corrosion values of the samples were 1.47 and $1.47(6.61 \%$ and $6.68 \%)$ and the difference values were 0.00 and $0.07 \%$, the coated samples were 1.37 and $1.38(-6.26 \%$ and $-6.23 \%)$ and the difference value of $0.01 \%$ and $0.06 \%$. The maximum computed ultimate tensile strength percentage for comparison controlled $-6.61 \%$ versus corroded $6.68 \%$ and closed $-6.23 \%$, and different peaks controlled $0.06 \%$, corroded $0.07 \%$, and coated $0.06 \%$, as stated by ([10], [11], [16], [12]). From the calculation results 
obtained, summarized in Tables 3.4 and 3.5 and displayed graphically in Figures 3.1 - 3.8, the yield strength, tensile strength, and deformation ratio of the mean, percentile and controlled differential potential values, uncoated (corroded) and layered concrete slab samples were determined., coated samples had higher breaking loads compared to corroded samples with reduced breakdown load and low load-bearing capacity and with averageand percentile values in relation to the reference range, whereas uncoated (corroded) samples had a loadbearing capacity which is low and a reduced value compared to the reference range. The comparison results show that the low load carrying capacity is caused by the effect of corrosion attack on the uncoated (corroded) elements, which damage the reinforcing steel fibers, ribs, and passive formation and surface modification. The observed averagevalues for the coated samples were associated with the corrosion resistivity potential to penetrate the reinforcing steel with the formation of a protective membrane; This attribute indicates the efficiency and effectiveness of the exudate/resin as an inhibitor against corrosive effects of reinforced concrete structures exposed to the edges of strong, high salinity marine areas.

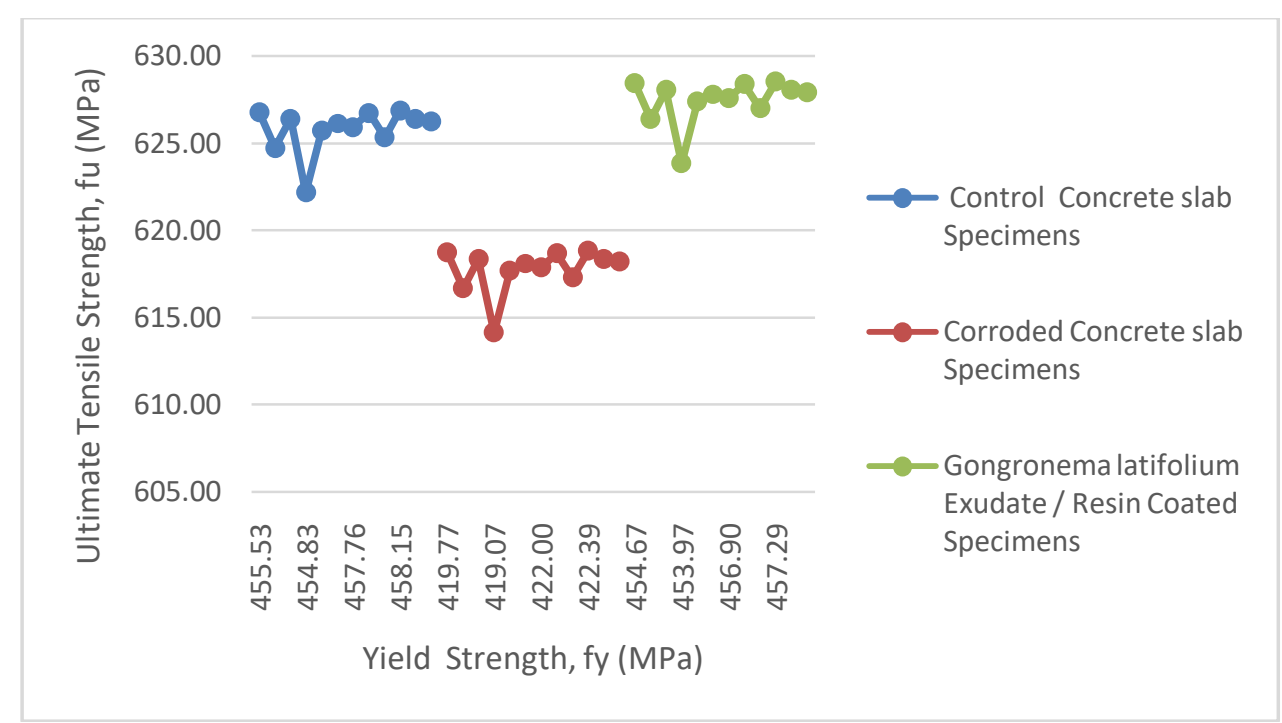

Figure 3.2 : Yield Strength versus Ultimate strength

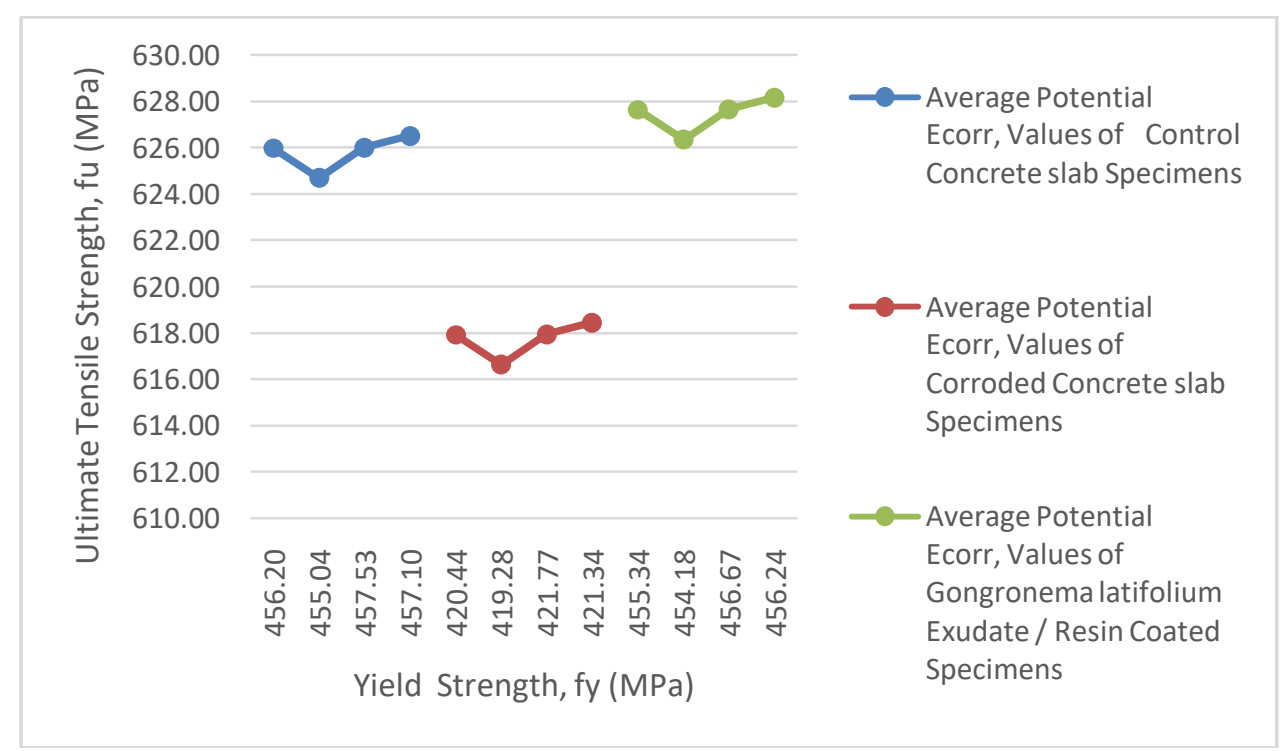

Figure 3.2A: Average Yield Strength versus Ultimate Tensile Strength 


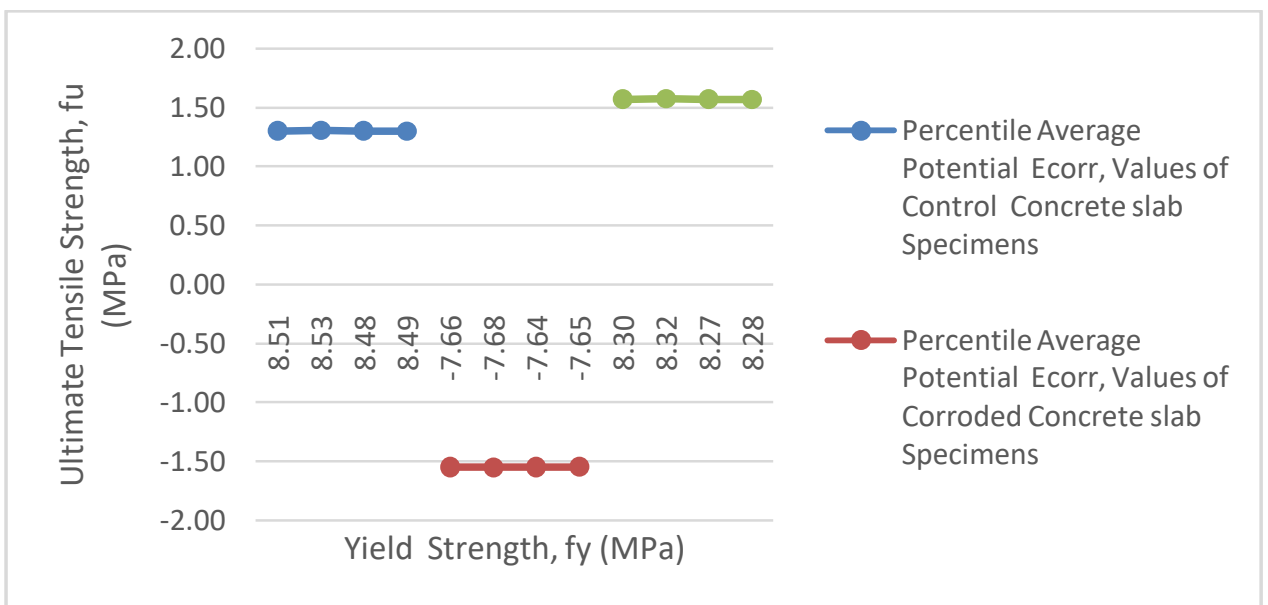

Figure 3.2B: Average Percentile Yield Strength versus Ultimate Tensile Strength

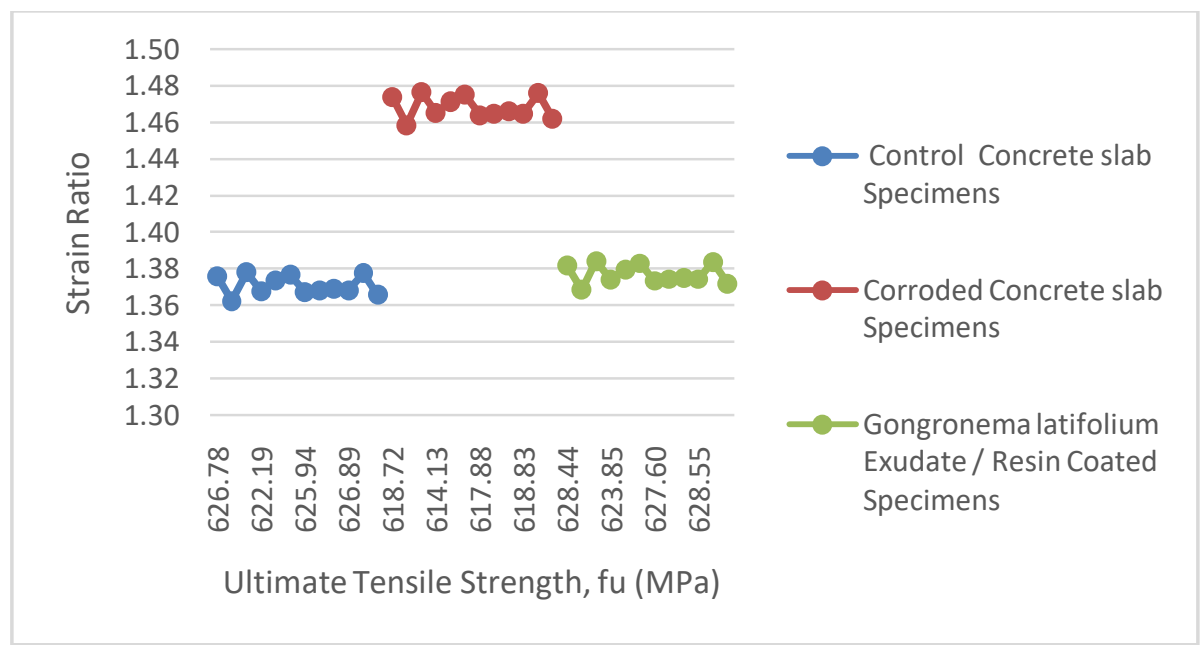

Figure 3.3: Ultimate Tensile Strength versus Strain Ratio

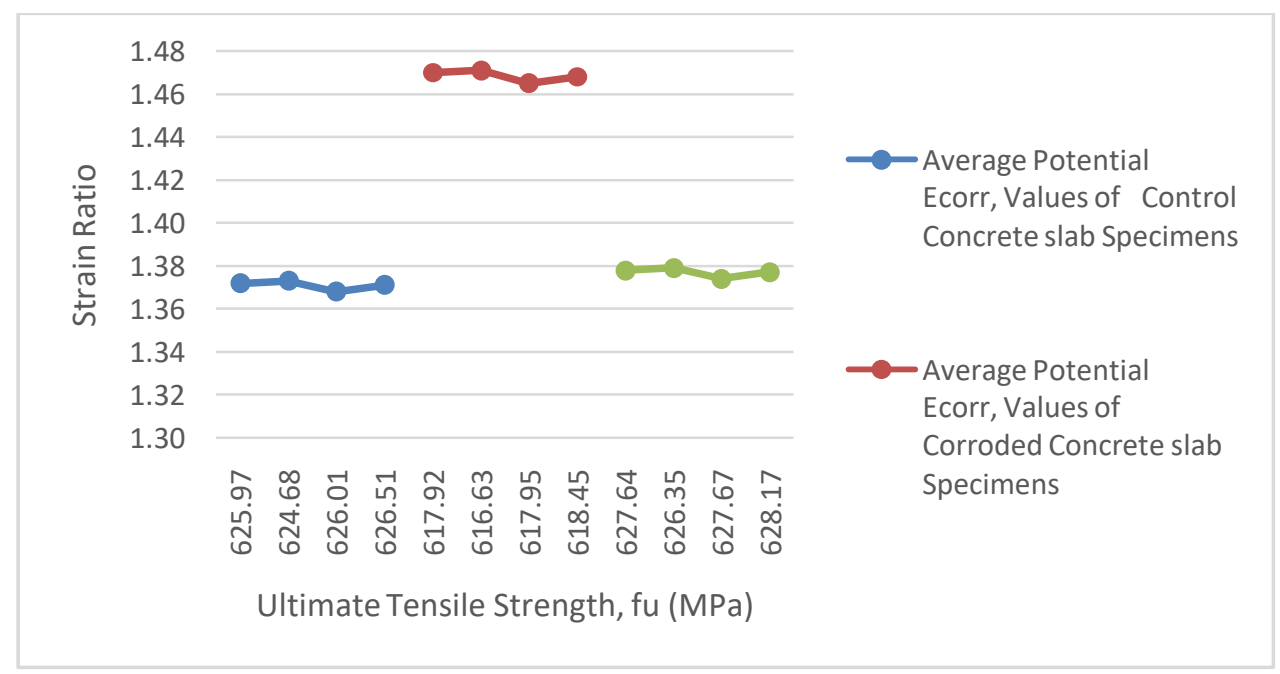

Figure 3.3A: Average Ultimate Tensile Strength versus Strain Ratio 


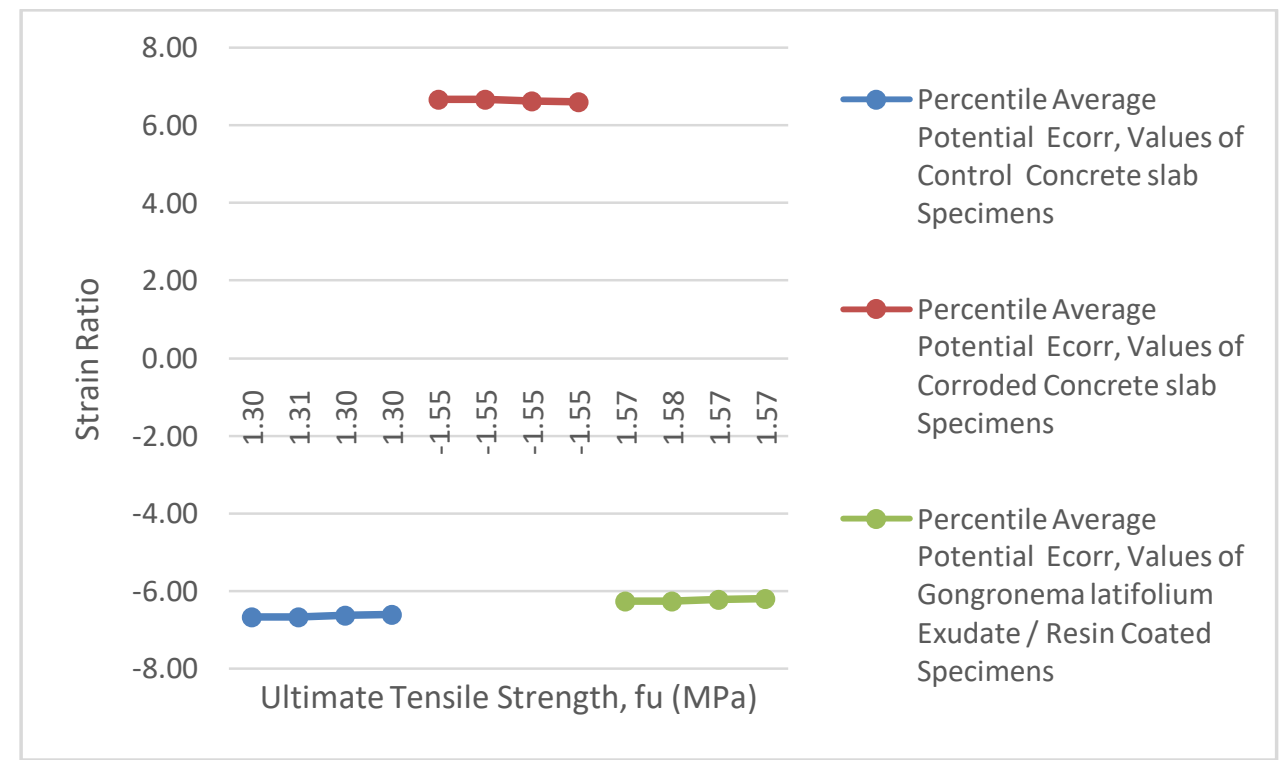

Figure 3.3B: Average percentile Ultimate Tensile Strength versus Strain Ratio

\subsection{Results of Mechanical Properties of Rebar Diameter, Cross-Sectional Area and Weight Loss / Increase of Embedded Reinforcing Steel in Concrete Slab}

Corrosion creates tensile stresses in the reinforcement, leading to early cracking and poor construction life. It is known that chloride is incorporated into concrete from various sources ([22], [23]), that concrete containing chloride ions from seawater and aggregates can be used as an accelerator. Global environmental concerns are increasing and are likely to influence the choice of corrosion inhibitors in the future. Inorganic inhibitors and more environmentally friendly inhibitors exhibit high and environmentally friendly substances, non-toxic, generally widespread, and inexpensive for future use, based on these properties, there is a great demand for organic-based green inhibitors due to their biodegradable properties.

The rebar diameter before testing $(\mathrm{mm})$ the averageand the minimum and maximum percentile controlled $11.96 \mathrm{~mm}$ and $11.97 \mathrm{~mm}(0.416 \%$ and $0.419 \%)$ with a difference of 0.01 $\mathrm{mm}$ and $0.003 \%$, the corrosion value of the sample was $11.96 \mathrm{~mm}$ and $11.96 \mathrm{~mm}(0.417 \%$ and $0.42 \%$ ) and the difference values of $0.00 \mathrm{~mm}$ and $0.003 \%$ and the coated sample values were $11.96 \mathrm{~mm}$ and $11.96 \mathrm{~mm}(0.411 \%$ and 0.42$) \%$ and the difference computed values of $0.00 \mathrm{~mm}$ and $0.009 \%$ ) The weight of the pieces of reinforcement before the corrosion test shows a smaller difference in relation to the product and form of the company, as well as by-products used in the production process and the maximum values obtained are the mean, percentile and difference in diameter of the reinforcement after corrosion $(\mathrm{mm})$ for the controlled sample were $11.96 \mathrm{~mm}$ and $11.97 \mathrm{~mm}(0.416 \%$ and $0.419 \%)$ with a difference of $0.01 \mathrm{~mm}$ and $0.003 \%$, with $100 \%$ of the reference values maintained, the values for the corroded sample were $11.91 \mathrm{~mm}$ and $11.92 \mathrm{~mm}(-0.99 \%$ and $-0.88 \%)$ and the difference between 0.01 $\mathrm{mm}$ and $0.106 \%$, the value of the sample coated are $12.02 \mathrm{~mm}$ and $12.02 \mathrm{~mm}(0.128 \%$ and $0.122 \%$ ) and the difference is $0.00 \mathrm{~mm}$ and $0.014 \%$. The maximum computed percentage was controlled from $0.419 \%$ versus $-0.88 \%$ corroded and $0.142 \%$ coated, with the difference in the percentage corroded being $0.106 \%$ versus $0.014 \%$ coated. The results obtained in Tables 3.4 and 3.5, which are summarized in Tables 3.1, 3.2, and 3.3 and shown graphically in Figures 3.3-3.6b, show the effect of corrosion attack on reinforcing steel embedded in the concrete slab, which is exposed to induced corrosion-accelerating activity. For comparison, 
the results of the corroded samples showed a reduction and reduction value compared to the diameter of the reinforcement before and after the induction accelerated corrosion test with a percentage reduction in value from $0.419 \%$ to $-0.88 \%$ and an average value from $11.97 \mathrm{~mm}$ to $11.92 \mathrm{~mm}$.

The decrease/increase (diameter) of cross-sectional area Minimum and maximum averageand percentile values were checked up to $100 \%$, with no decrease or increase after 360 days of immersion in freshwater. Corroded sample values were $0.007 \mathrm{~mm}$ and $0.059 \mathrm{~mm}(-22.03 \%$ and $-22.03 \%$ ) and the difference in corrosion was $0.0 \mathrm{~mm}$ and $0.00 \%$, coated sample values were $0.065 \mathrm{~mm}$ and $0.071 \mathrm{~mm}(28.26 \%$ and $28.26 \%)$ and the difference between $0.006 \mathrm{~mm}$ and $0.00 \%$. The relative averageand difference in percentage values of decrease/increase (diameter) of cross-sectional area between coated and corroded samples ranged from $28.26 \%$ to $-22.03 \%$. The decrease in average and percentilesvalues indicated that the corrosion effect led to a reduction in diameter and cross-sectional area, fiber degradation, rib reduction, and surface modification, whereas elements coated with exudate/resin confirmed an increase in volume as the layer thickness difference as stated by ([10], [16], [12], [11]). In summary, it can be said that the exudate/resin has inhibitory properties against corrosive effects on reinforcing steel embedded in the concrete slab sample, which is induced in an environment with high salt content.

The values of rebar unit weight - before Test $(\mathrm{kg})$, the average and percentilesof minimum, maximum, and differential of the controlled sample were $0.882 \mathrm{~kg}$ and $0.888 \mathrm{~kg}(9.78 \%$ and $9.99 \%$ ), and the difference was $0.006 \%$ and $0.21 \%$. the corroded samples weighed $0.88 \mathrm{~kg}$ and $0.891 \mathrm{~kg}(-17.9 \%$ and $-15.6 \%)$ and the difference was $0.011 \%$ and $2.32 \%$, the coated samples were $0.88 \mathrm{~kg}$ and $0.891 \mathrm{~kg}(13.93 \%$ and $17.13 \%)$ with a difference of $0.011 \%$ and $3.2 \%$. The average and percentilesof reinforcement weight after corrosion $(\mathrm{Kg})$ and the aggregate difference values of the minimum and maximum values of the controlled samples were $0.88 \mathrm{~kg}$ and $0.88 \mathrm{~kg}(0.00 \%$ and $0.00 \%)$ and the difference was $0.00 \%$ and $0.00 \%$, corroded samples $0.83 \mathrm{~kg}$ and $0.835 \mathrm{~kg}-12.6 \%$ and $-12.6 \%$ ) and the difference of $0.005 \%$ and $0.03 \%$, the value of coated samples was $0.945 \mathrm{~kg}$ and $0.955 \mathrm{~kg}(14.41 \%$ and $14.44 \%)$ and the difference between $0.01 \%$ and $0.03 \%$. Average and percentage losses/gains of minimum and maximum units of steel $(\mathrm{kg})$ and percentage differences in comparison controlled as a result of aggregation in freshwater tanks with no trace of corrosion potential relative to the corroded sample, values maintained at $100 \%$, and values of $0.052 \mathrm{~kg}$ and $0.061 \mathrm{~kg}(-24.7 \%$ and $-23.5 \%)$ and coverage of $0.061 \mathrm{~kg}$ and $0.079 \mathrm{~kg}(34.06 \%$ and $39.35 \%)$. The calculation results from Tables 3.1-3.3 and in 3.4 - 3.5 are summarized and plotted graphically in Figure 3.7-3.87, listing the effects of corrosion on uncoated (corroded) and coated reinforcing steel and checking the weight of the pieces of reinforcement before and after testing for corrosion and settlement. /weight gain. For comparison, the results obtained show a reduction/reduction and reduction of averageand percentile values for coatings with $0.955 \mathrm{~kg}$ to $0.835 \mathrm{~kg}$ and $14.44 \%$ to $-12.6 \%$ corroded, as stated by ([10], [16], [12], [11]). The combined results show that the corrosion effect on the corroded samples causes a decrease/decrease in weight compared to coatings with percentile loading and an increase in averagevalues, which causes a slight increase in volume with coating thickness. This study shows the efficacy and effectiveness of exudate/resin as an inhibitor against the effects of corrosion on reinforcement embedded in samples of concrete slabs exposed to induced corrosion. 


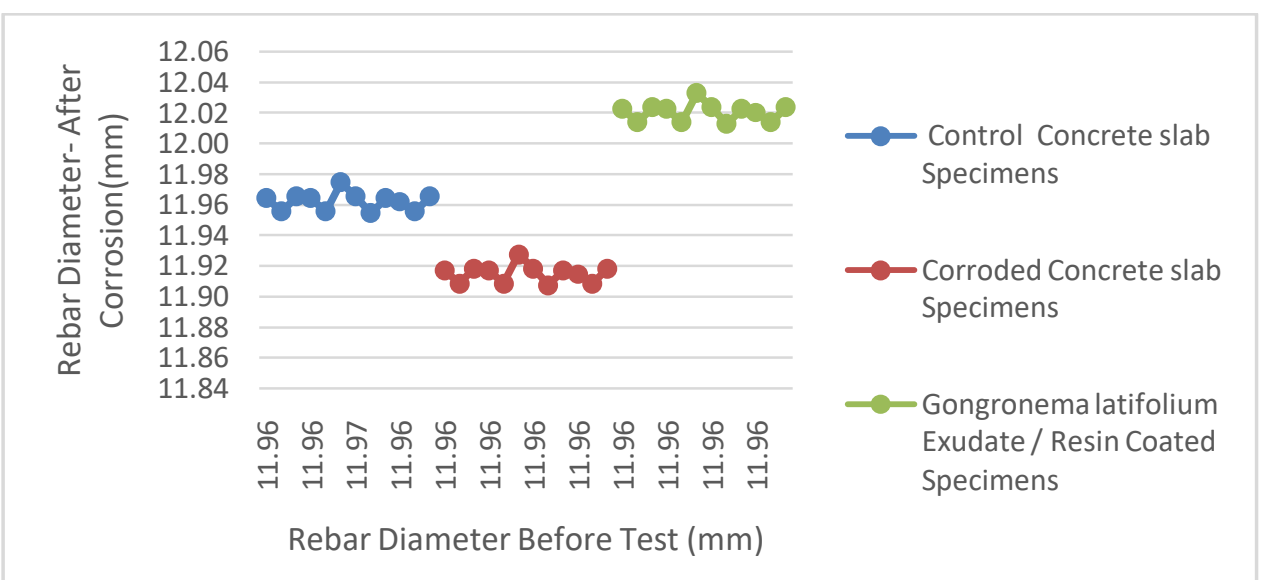

Figure 3.4: Rebar Diameter Before Test $(\mathrm{mm})$ versus Rebar Diameter- After Corrosion(mm)

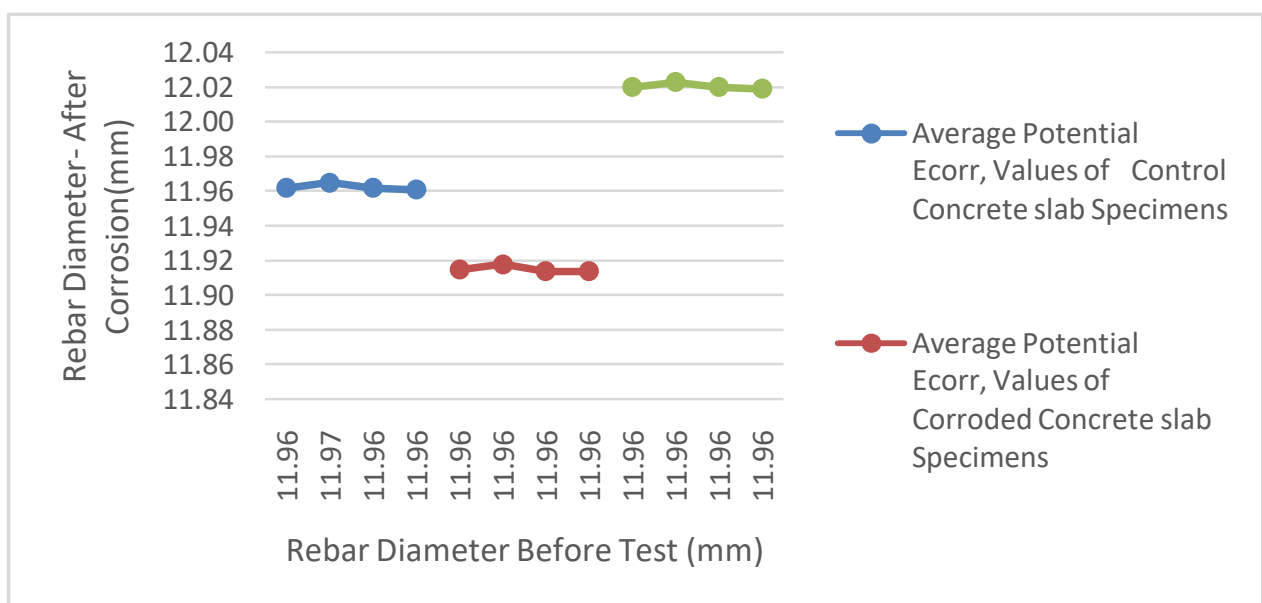

Figure 3.4B: Average Percentile Rebar Diameter Before Test $(\mathrm{mm})$ versus Rebar Diameter- After Corrosion(mm)

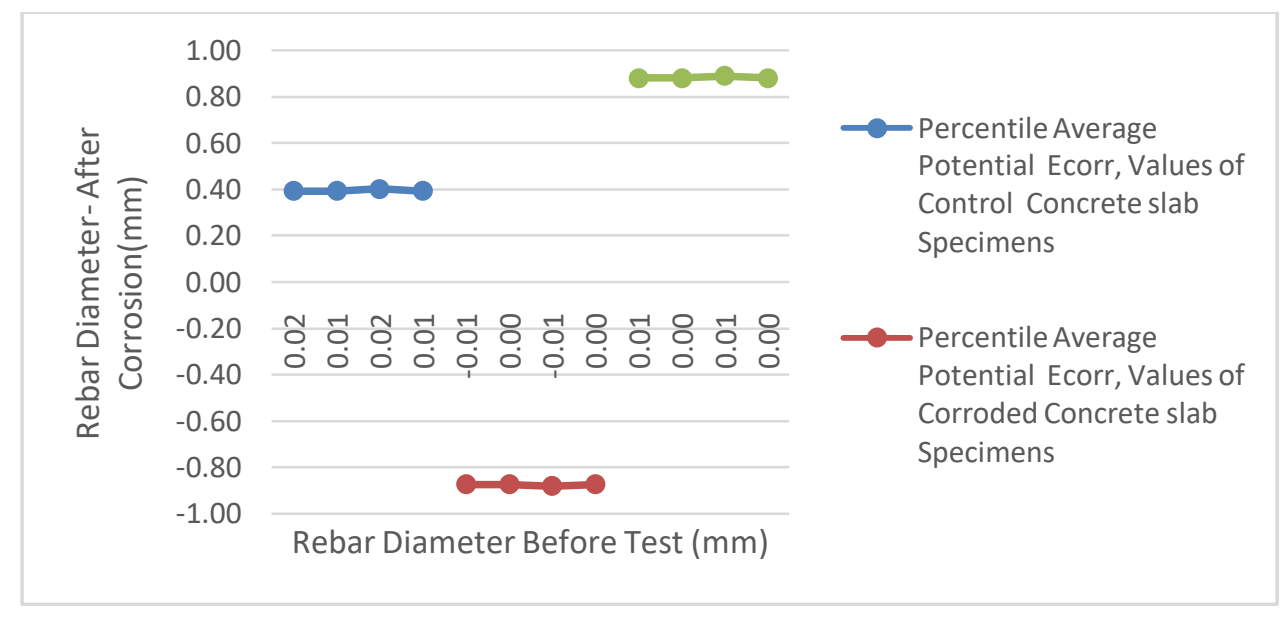

Figure 3.4A: Average Rebar Diameter Before Test $(\mathrm{mm})$ versus Rebar Diameter- After Corrosion(mm) 


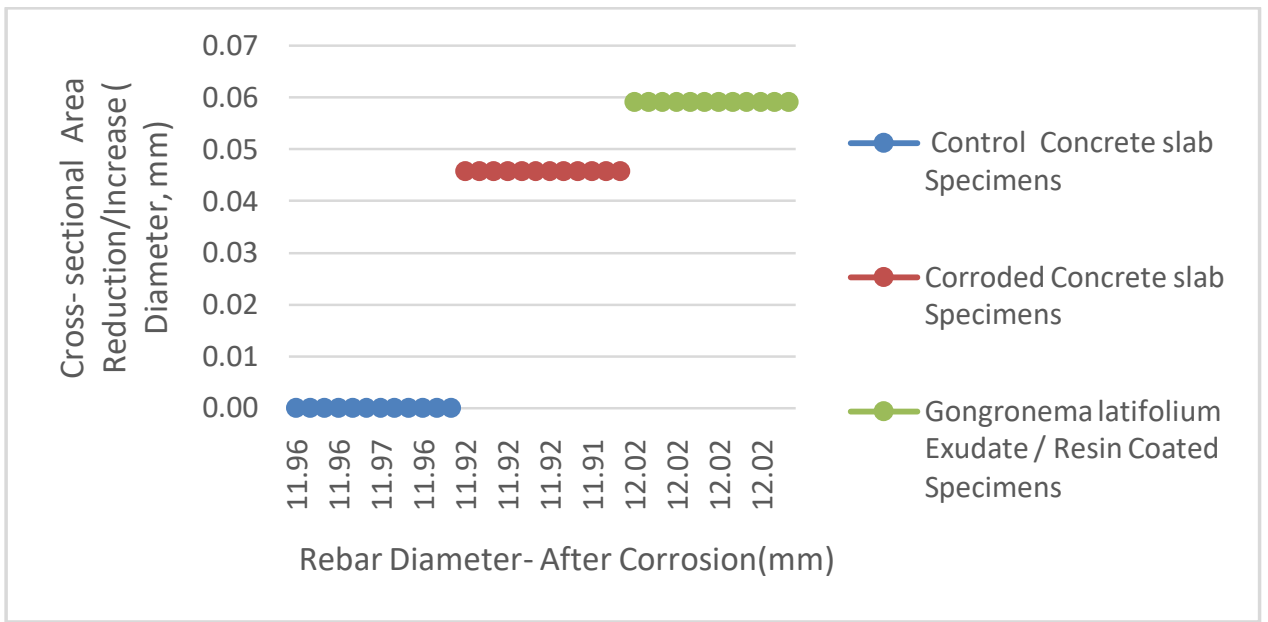

Figure 3.5: Rebar Diameter- After Corrosion(mm) versus Cross- section Area Reduction/Increase ( Diameter, mm

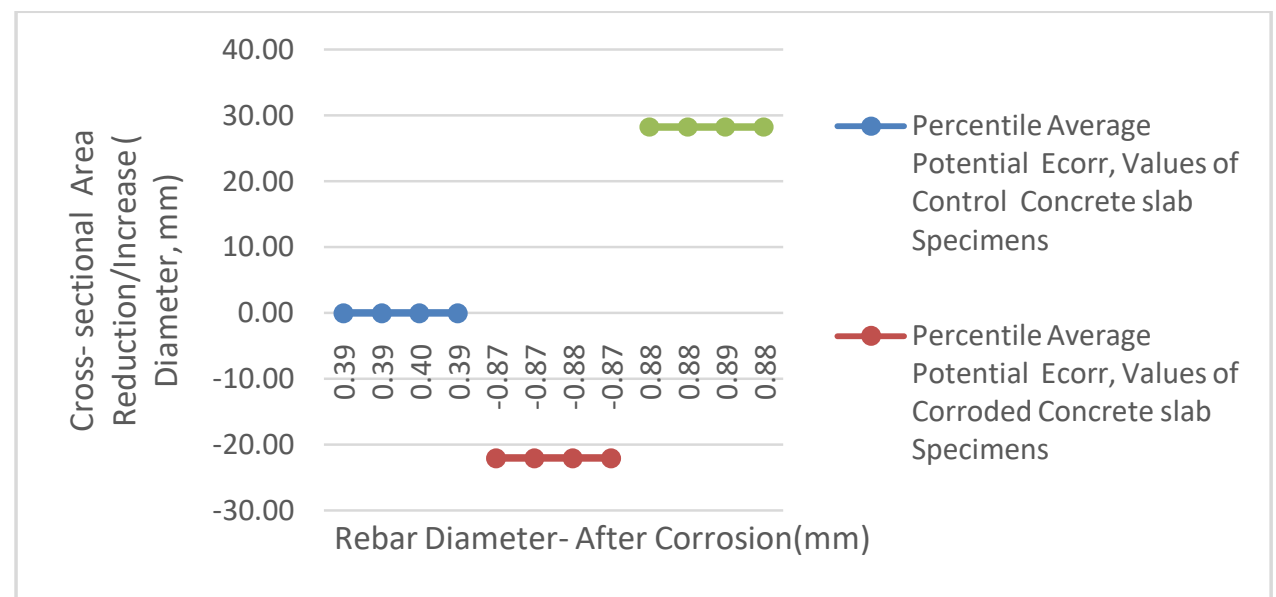

Figure 3.5A: Average Rebar Diameter- After Corrosion(mm) versus Cross- section Area Reduction/Increase ( Diameter, mm)

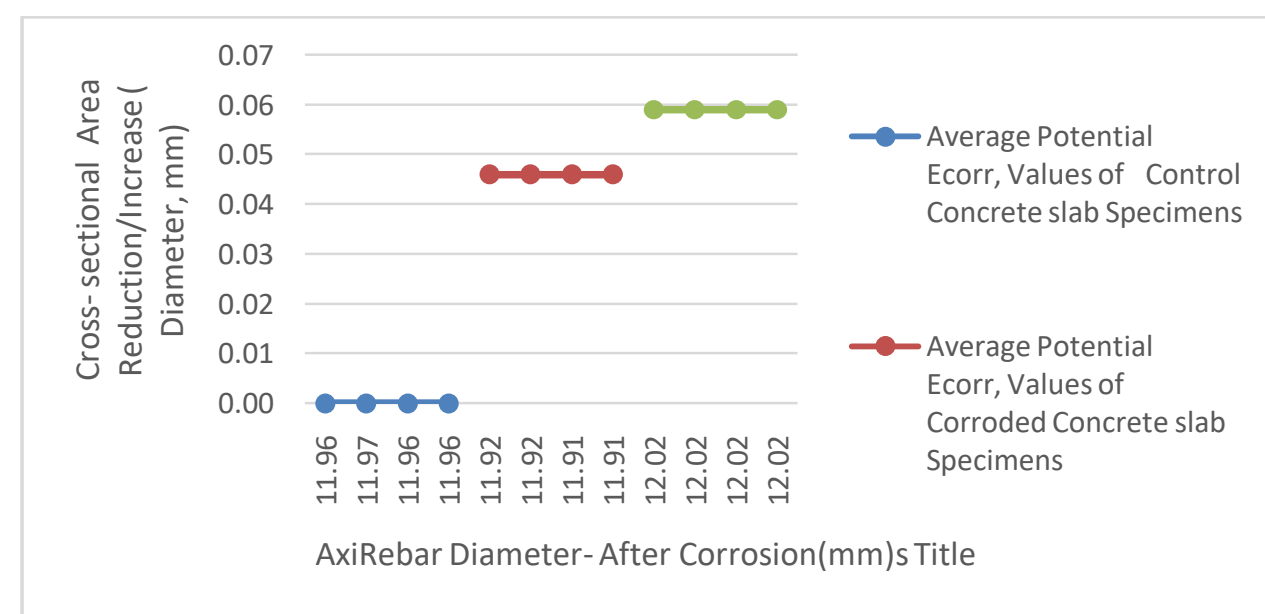

Figure 3.5B: Average Percentile Rebar Diameter- After Corrosion( $\mathrm{mm})$ versus Cross- section Area Reduction/Increase ( Diameter, mm) 


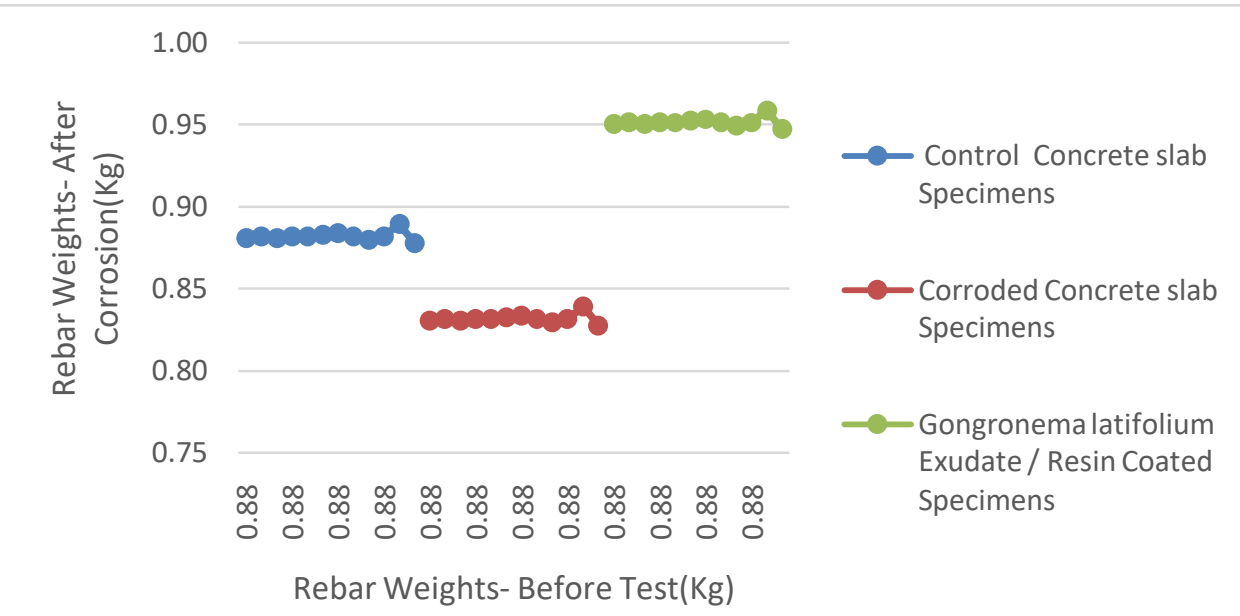

Figure 3.6: Rebar Weights- Before Test $(\mathrm{Kg})$ versus Rebar Weights- After Corrosion $(\mathrm{Kg})$

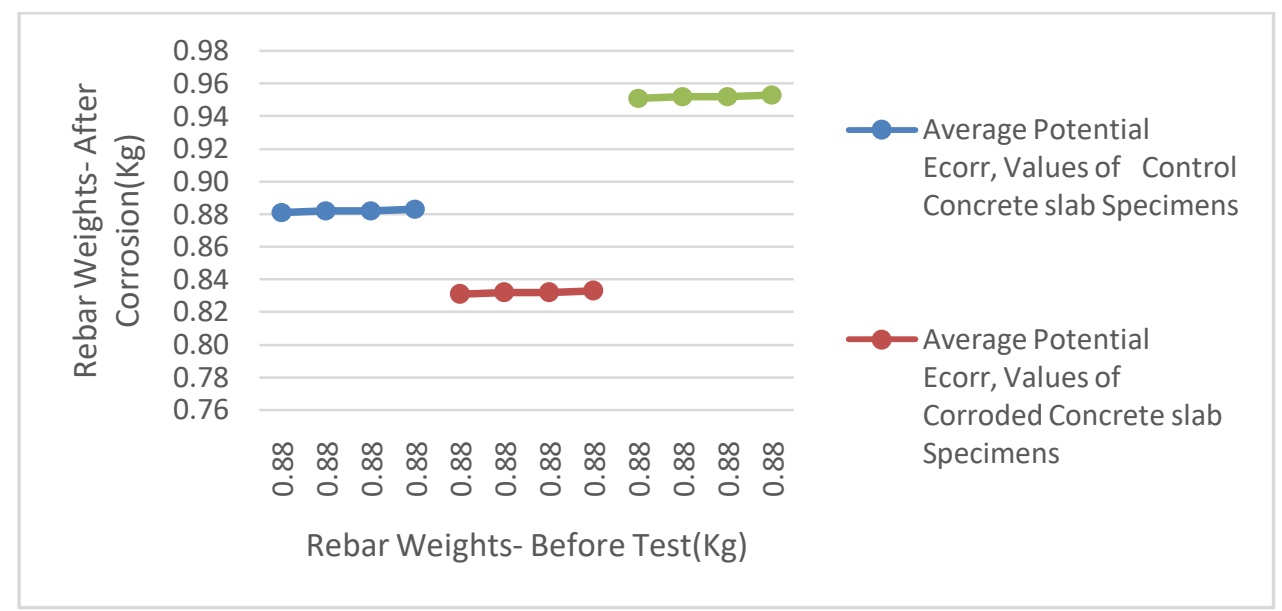

Figure 3.6A: Average Rebar Weights- Before Test $(\mathrm{Kg})$ versus Rebar WeightsAfter Corrosion $(\mathrm{Kg})$

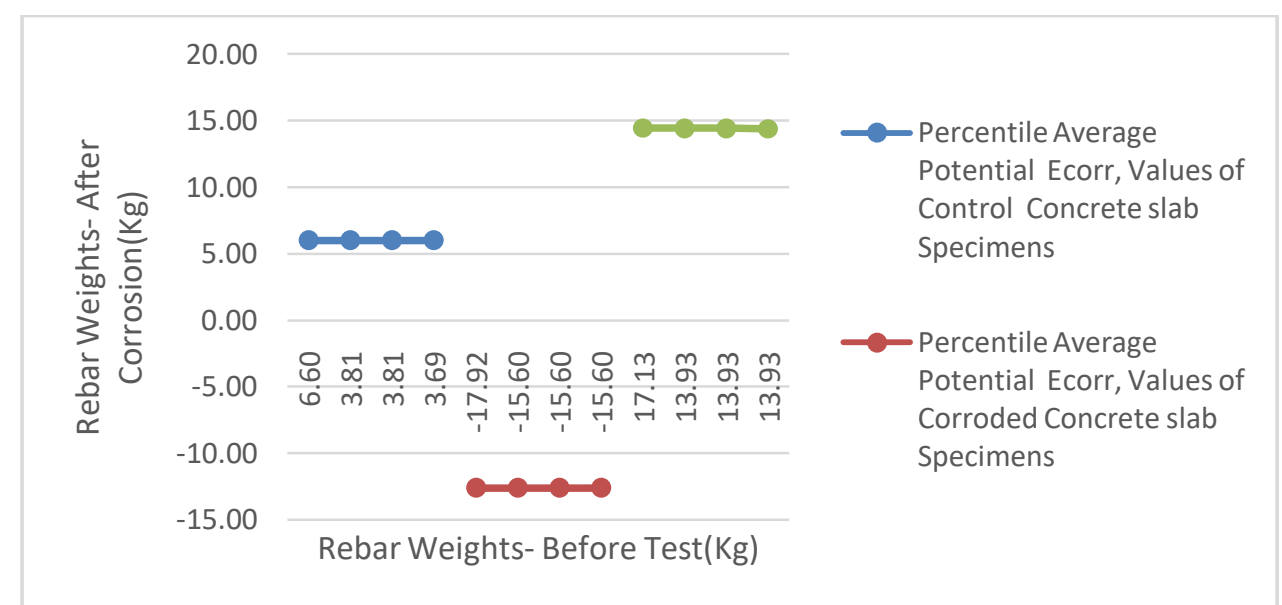

Figure 3.6B: Average Percentile Rebar Weights- Before Test $(\mathrm{Kg})$ versus Rebar WeightsAfter Corrosion $(\mathrm{Kg})$ 


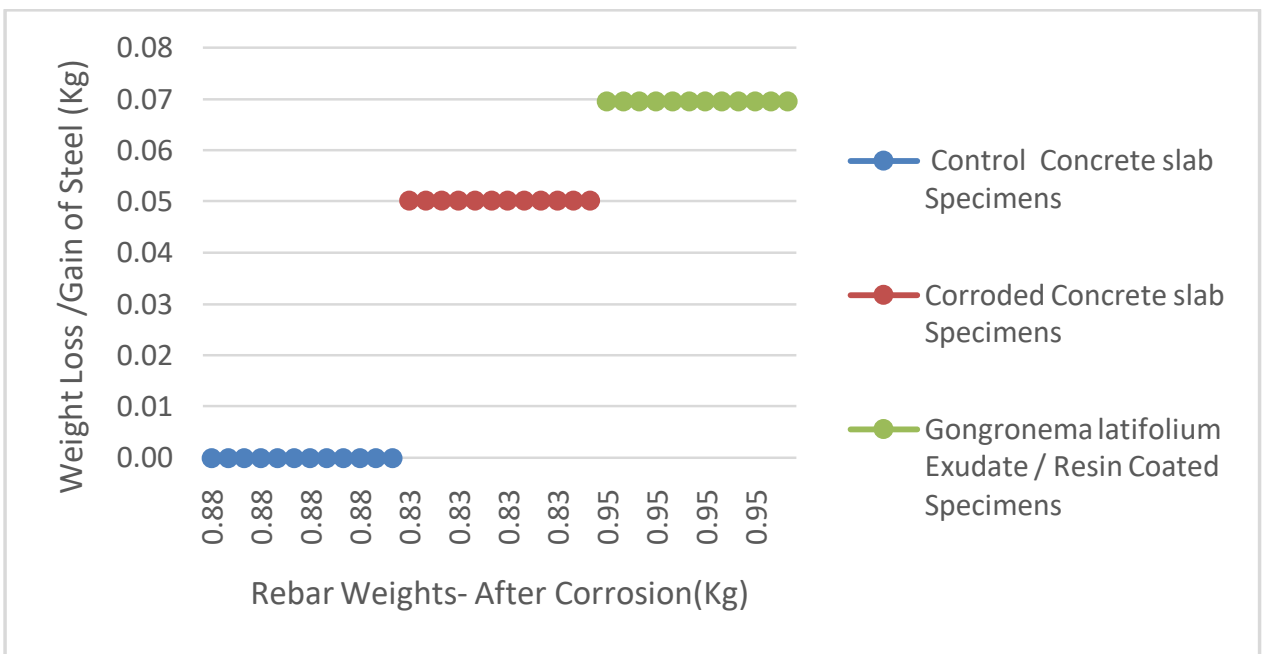

Figure 3.7: Rebar Weights- After Corrosion $(\mathrm{Kg})$ versus Weight Loss /Gain of Steel $(\mathrm{Kg})$

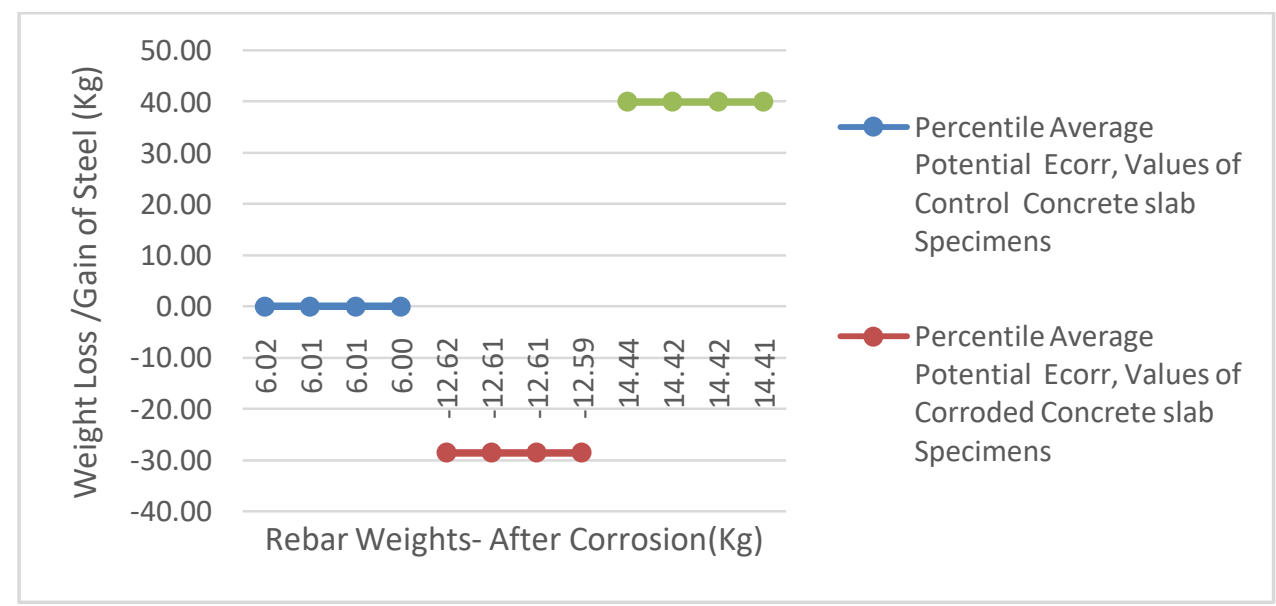

Figure 3.7A: Average Rebar Weights- After Corrosion(Kg) versus Weight Loss /Gain of Steel (Kg)

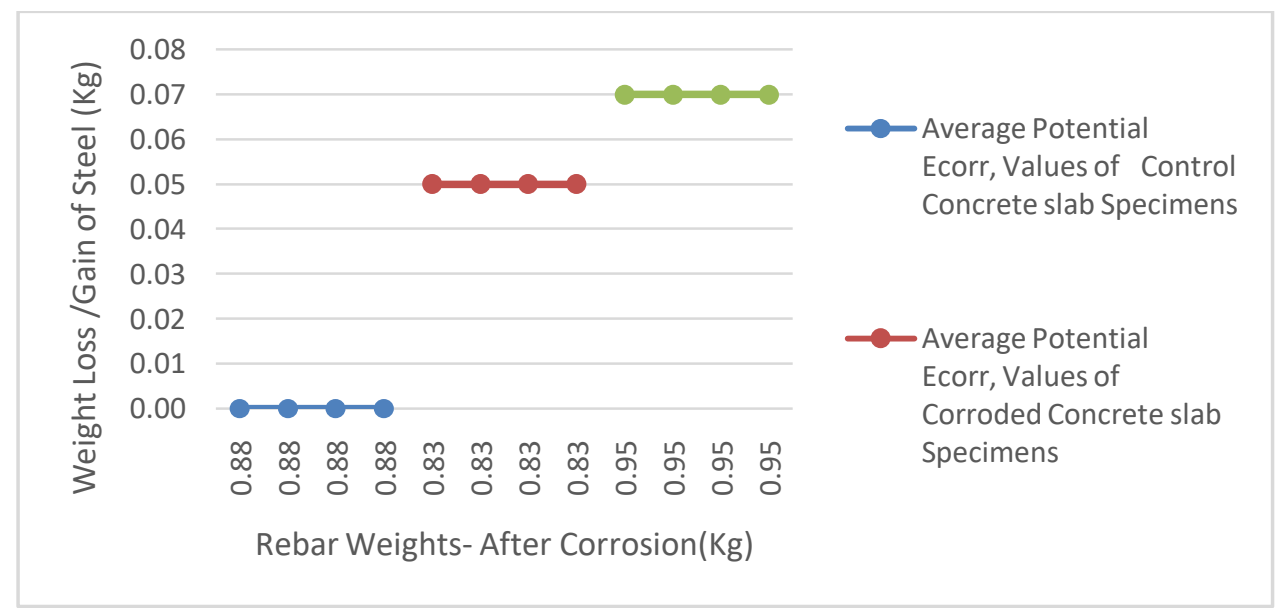

Figure 3.7B: Average Percentile Rebar Weights- After Corrosion( $\mathrm{Kg})$ versus Weight Loss /Gain of Steel (Kg) 


\section{CONCLUSION}

Experimental results showed the following conclusions:

i. Coated reinforcing steel showed no indications of corrosion presence

ii. Gongronema latifolium exudates / resins showed an inhibitory properties against corrosion attacks

iii. Reduction in diameter and cross-sectional areas were noticed in corroded samples

iv. Weight loss was witnessed in corroded samples while inhibited samples exhibited minute volumetric increase.

v. Yield strength and ultimate tensile strength reduction was noticed in corroded samples resulting from corrosion effect

\section{REFERENCES}

1) C. Andrade, and R. Andrea. Electrical Resistivity as Microstructural Parameter for Modelling of Service Life of Reinforced Concrete Structures. 2nd International Symposium on Service Life Design for Infrastructure, Delft, Netherlands, (October), 379388, 2010.

2) P. Domone, and J. Illston. Construction Materials: Their Nature and Behavior. CRC Press, Taylor and Francis, London, UK. 2010.

3) C. Andrade, M. Keddam, M. Novoa, X. Perez, M. Rangel, C., and H. Takenouti. Electrochemical Behaviour of Steel Rebars in Concrete: Influence of Environmental Factors and Cement Chemistry. Electrochimica Acta, no.46, pp. 3965-3972, 2018.

4) M. Saremi, and E. Mahallati. A Study on Chloride-Induced Depassivation of Mild Steel in Simulated Concrete Pore Solution. Cement and Concrete Research, no.32, pp. 19151921, 2002.

5) L. Bertolini, B. Elsener, P. Pedeferri, and R. Polder. Corrosion of steel in concrete: Prevention, Diagnosis, Repair. Wiley-VCH, Weinheim, 2004.

6) Z. Lounis, J. Zhang, and L. Daigle, L. Probabilistic Study Chloride-Induced Corrosion of Carbon Steel in Concrete Structures. 9th ASCE Joint Specialty Conference on Probablistic Mechanisms and Structural Reliability, Albuquerque, New Mexico, 1-6, 2004.

7) B. Elsener. Corrosion Rate of Steel in Concrete Measurements beyond the Tafel Low. Corrosion Science, no.47, pp. 3019-3033, 2005.

8) R. M. Schroeder, and I. L. Muller, I. L. Stress Corrosion Cracking and Hydrogen Embitterment Susceptibility of an Eutectoid Steel Employed in Prestressed Concrete. Corrosion Science, no.45, pp. 1969-1983, 2003.

9) S. Ramadan, L. Gaillet, C. Tessier, and H. Idrissi. Detection of Stress Corrosion Cracking of High-Strength Steel Used in Prestressed Concrete Structures By Acoustic Emission Technique. Applied Surface Science, no.254, pp. 2255-2261, 2008.

10) K. Charles, A. Bright, and P. G. Irimiagha. Investigation on Mechanism of Steel Bar Corrosion of Reinforced Concrete Structures in Aqueous Solution Using Wenner Technique. International Journal of Scientific \& Engineering Research, vol.9, no. 4, pp. $1731-1748,2018$.

11) K. Charles, B. Nwinuka, and K. F. O. Philip. Investigation of Corrosion Probability Assessment and Concrete Resistivity of Steel Inhibited Reinforcement of Reinforced Concrete Structures on Severe Condition. International Journal of Scientific and Engineering Research, vol.9, no.4, pp. 1714 -1730, 2018.

12) Petaba, L. D., Charles, K., Kanee, S., "Electrochemical Corrosion Measurement of NonInhibited and Inhibited Reinforcement Mechanical Properties Embedded in Concrete. International Journal of Scientific and Engineering Research, Vol. 10, no.9, pp. 1180 1196, 2019. 
13) V. Novokshcheov. Salt penetration and corrosion in pre-stressed concrete member. Washington, D. C., Federal Highway, 2000.

14) A. Skotinck. Corrosion of concrete and its prevention," 6th International Conference on Corrosion Moscow, Russia: pp.18-25, 2000.

15) J. Slater. Corrosion of Metals in Association with Concrete. New Jersey Prentice-Hall Inc. Stem M and Geary AL. Electrochemical polarization: a theoretical analysis of the shape of polarization curves, Journal of the Electrochemical Society, no.104, pp. 56-63, 2001.

16) BS 882; - Specification for aggregates from natural sources for concrete, British Standards Institute. London, United Kingdom, 1992.

17) BS EN 196-6; - Methods of Testing Cement, "Determination of fineness, British Standards Institute. London, United Kingdom, 2010.

18) BS 12390-5; - Testing Hardened Concrete: Flexural Strength Test of Specimens, British Standards Institute. London, United Kingdom, 2005.

19) BS 4449:2005+A3 -Steel for Reinforcement of Concrete. British Standards Institute. London, United Kingdom, 2005.

20) K. R. Gowers and S. G. Millard. Electrochemical techniques for corrosion assessment of reinforced concrete structures. Structures and Building. Vol. 134, Issue 2, pp 129 - 137, 1999a.

21) J.W. Figg and A. F. Marsden, "Development of inspection techniques for reinforced concrete: a state of the art survey of electrical potential and resistivity measurements In Concrete in the Oceans, HMSO, London," Technical Report 10, OHT 84 205, 1985

22) M. Stem and A. L. Geary. Electrochemical Polarization: A Theoretical Analysis of the Shape of Polarization curves. Journal of the Electrochemical Society, no.104, pp. 56-63, 1957.

23) ASTM Standard C876 - Standard test method for corrosion potentials of uncoated reinforcing steel in concrete, A. International, Editor. 2012, ASTM International: West Conshohocken, PA, 2012.

24) ASTMC876-91: - Standard Test Method for Half-cell Potentials of Uncoated Reinforcing Steel in Concrete, 1999.

25) W. Morris, A. Vico, and M. Vazquez. Chloride induced corrosion of reinforcing steel evaluated by resistivity measurements. Electrochimica Acta, Vol. 49, pp. 4447-4453, 2004.

26) K.Y. Ann, and H.W. Song. Chloride threshold level for corrosion of steel in concrete", Corrosion Science, no.49, pp. 4113-4133, 2007.

27) P. Langford and J. Broomfield. Monitoring the corrosion of reinforcing steel. Construction Repair, pp. 32-36, 1987.

28) D. Macdonald. Design Options for Corrosion Protection. 8th International Symposium," Australia, pp.75-83, 2003. 